\title{
The Effect of Boundary Slip on the Transient Pulsatile Flow of a Modified Second-Grade Fluid
}

\author{
N. Khajohnsaksumeth, ${ }^{1}$ B. Wiwatanapataphee, ${ }^{2}$ and $\mathrm{Y} . \mathrm{H}$. $\mathrm{Wu}^{1}$ \\ ${ }^{1}$ Department of Mathematics and Statistics, Curtin University, Perth, WA 6845, Australia \\ ${ }^{2}$ Department of Mathematics, Mahidol University, Faculty of Science, Bangkok 10400, Thailand
}

Correspondence should be addressed to N. Khajohnsaksumeth; jobscma@yahoo.com and B. Wiwatanapataphee; benchawan.wiw@mahidol.ac.th

Received 20 May 2013; Accepted 9 August 2013

Academic Editor: Rasajit Bera

Copyright (C) 2013 N. Khajohnsaksumeth et al. This is an open access article distributed under the Creative Commons Attribution License, which permits unrestricted use, distribution, and reproduction in any medium, provided the original work is properly cited.

\begin{abstract}
We investigate the effect of boundary slip on the transient pulsatile fluid flow through a vessel with body acceleration. The FahraeusLindqvist effect, expressing the fluid behavior near the wall by the Newtonian fluid while in the core by a non-Newtonian fluid, is also taken into account. To describe the non-Newtonian behavior, we use the modified second-grade fluid model in which the viscosity and the normal stresses are represented in terms of the shear rate. The complete set of equations are then established and formulated in a dimensionless form. For a special case of the material parameter, we derive an analytical solution for the problem, while for the general case, we solve the problem numerically. Our subsequent analytical and numerical results show that the slip parameter has a very significant influence on the velocity profile and also on the convergence rate of the numerical solutions.
\end{abstract}

\section{Introduction}

In this paper, we study a fluid-structure interaction problem, namely, the effect of boundary slip on the flow of a nonNewtonian fluid through microchannels. This problem has many applications, and in this paper we particularly focus on blood flow in the cardiovascular system.

For the study of blood flow in arteries, two major types of constitutive models have been used. The first type of models is based on the microcontinuum or the structured continuum theories [1-6] in which the balance laws are used to determine the characteristics of blood motion. In the other type of models, blood is considered as a suspension, and its flow is modeled by the non-Newtonian fluid mechanics. Due to the red blood cells (RBC) migration as shown experimentally, blood has been modeled as a two-stage fluid by many researchers [7-9]. The first stage is a peripheral layer which is modeled as a Newtonian viscous fluid, while the other one is a centre core which is modeled as a non-Newtonian fluid. The effect of body acceleration and pulsatile conditions were taken into account under the same problem by Majhi et al. $[7,10]$. Later, Massoudi and Phuoc [11] used the (generalized) second-grade fluid constitutive model to describe the shear thinning and normal stress effect, and the behavior of blood flow near the wall is modeled by the Newtonian fluid model, while the behavior of the blood flow at the core is described by the second-grade fluid model.

In all of the above mentioned models, the so-called noslip boundary condition is used; namely, the velocity of flow relative to the solid is zero on the fluid-solid interface [12]. Although the no-slip condition is supported by many experimental results, the existence of slip of a fluid on the solid surface was also observed by many other researches [13-20]. The Navier slip condition has been used by various researchers to describe boundary slip and is a more general boundary condition, in which the fluid velocity component tangential to the solid surface, relative to the solid surface, is proportional to the shear stress on the fluid-solid interface and the slip length. The surface characteristics constant, slip length, describes the "slipperiness" of the surface. Recently, 

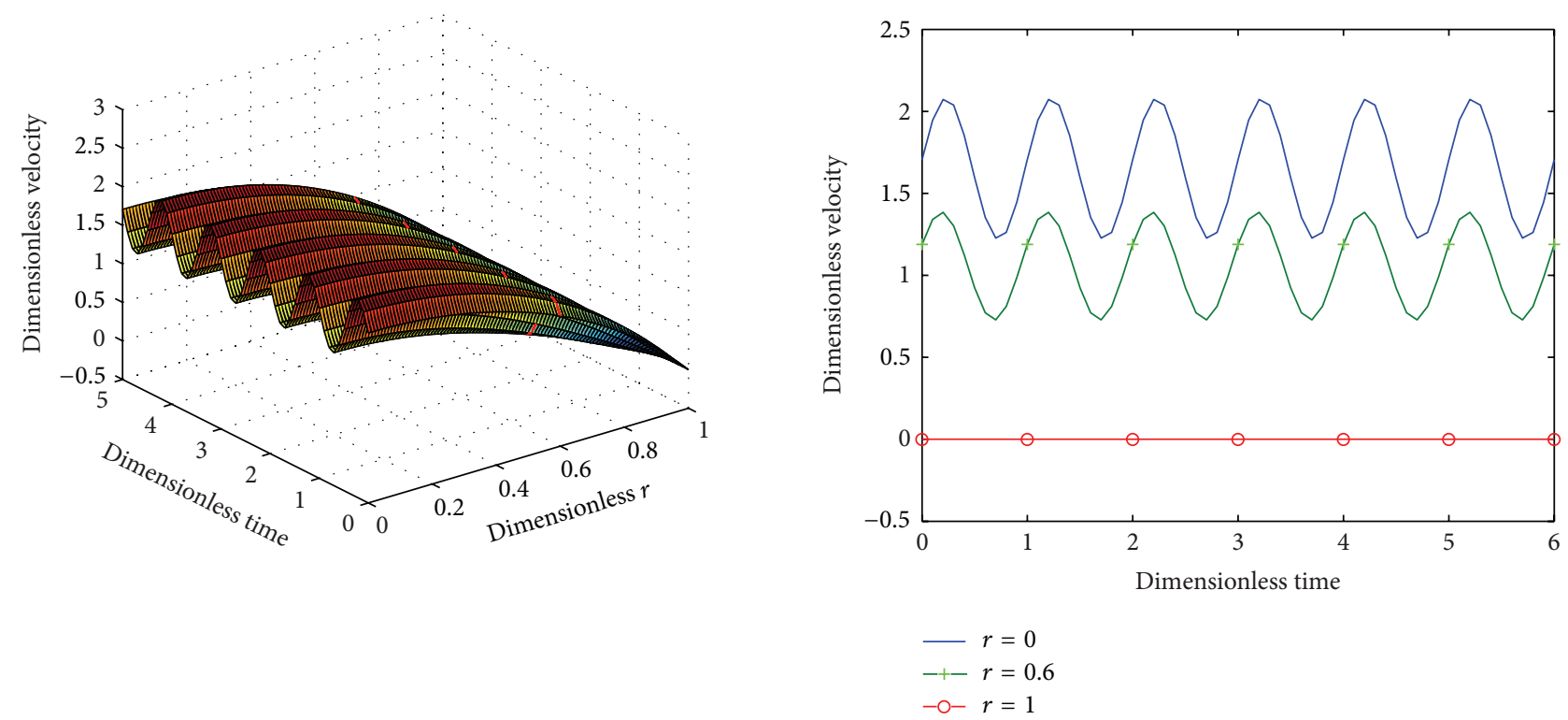

(a)
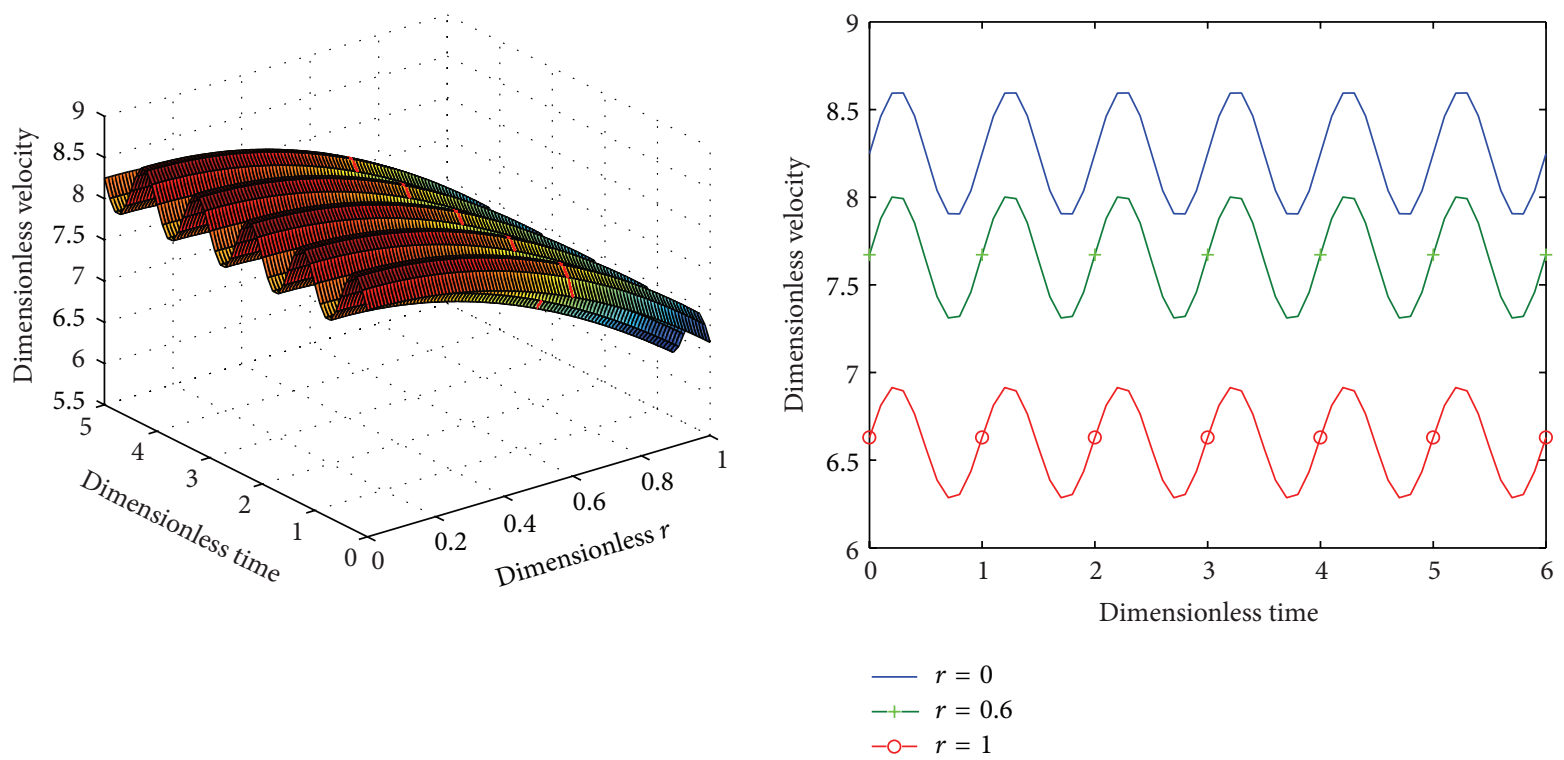

(b)

Figure 1: The velocity profile in the small artery with radius $0.15 \mathrm{~cm}$ under two different slip parameter values: (a) $l_{b}=0$; (b) $l_{b}=2$. In the figure, the $3 \mathrm{D}$ graphs show the variation of velocity as a function of time and location, while the $2 \mathrm{D}$ graphs show the variation of velocity with time at three radial locations including the artery centre $(r=0)$, the interface of inner-outer layer $(r=0.6)$, and the arterial wall $(r=1)$.

we and many other researchers have investigated various flow problems of Newtonian fluids with the traditional no-slip and the Navier slip boundary conditions [12, 20-30], and it is found that the boundary slip and the slip parameter have significant influence on the flow of Newtonian fluids through microchannels and tubes.

Motivated by the above mentioned work, we extend previous work on slip flows of Newtonian fluids [21, 22] to the case involving both Newtonian and non-Newtonian fluid flow in the flow region. The new feature and contribution of this work include establishment of the underlying boundary value problem for the problem, the derivation of an exact solution for a special case, and demonstration of the influence of the slip parameter on the flow profile and flow behavior. The rest of the paper is organized as follows. In Section 2, we present the underlying boundary value problem for the problem in dimensionless form. Then in Section 3, we derive an exact solution for a special case. In Section 4, we investigate numerically the effect of the slip parameter for the general case. Finally, a conclusion is given in Section 5. 

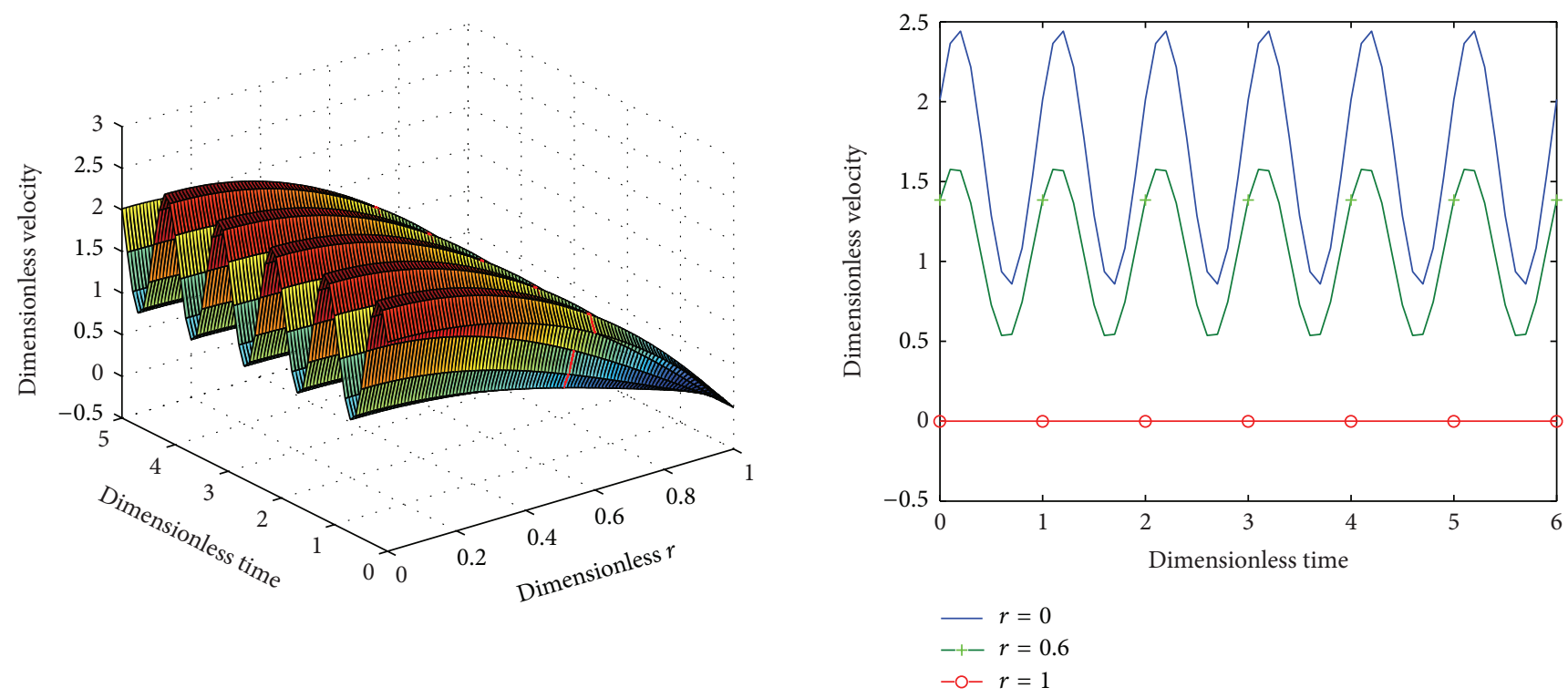

(a)
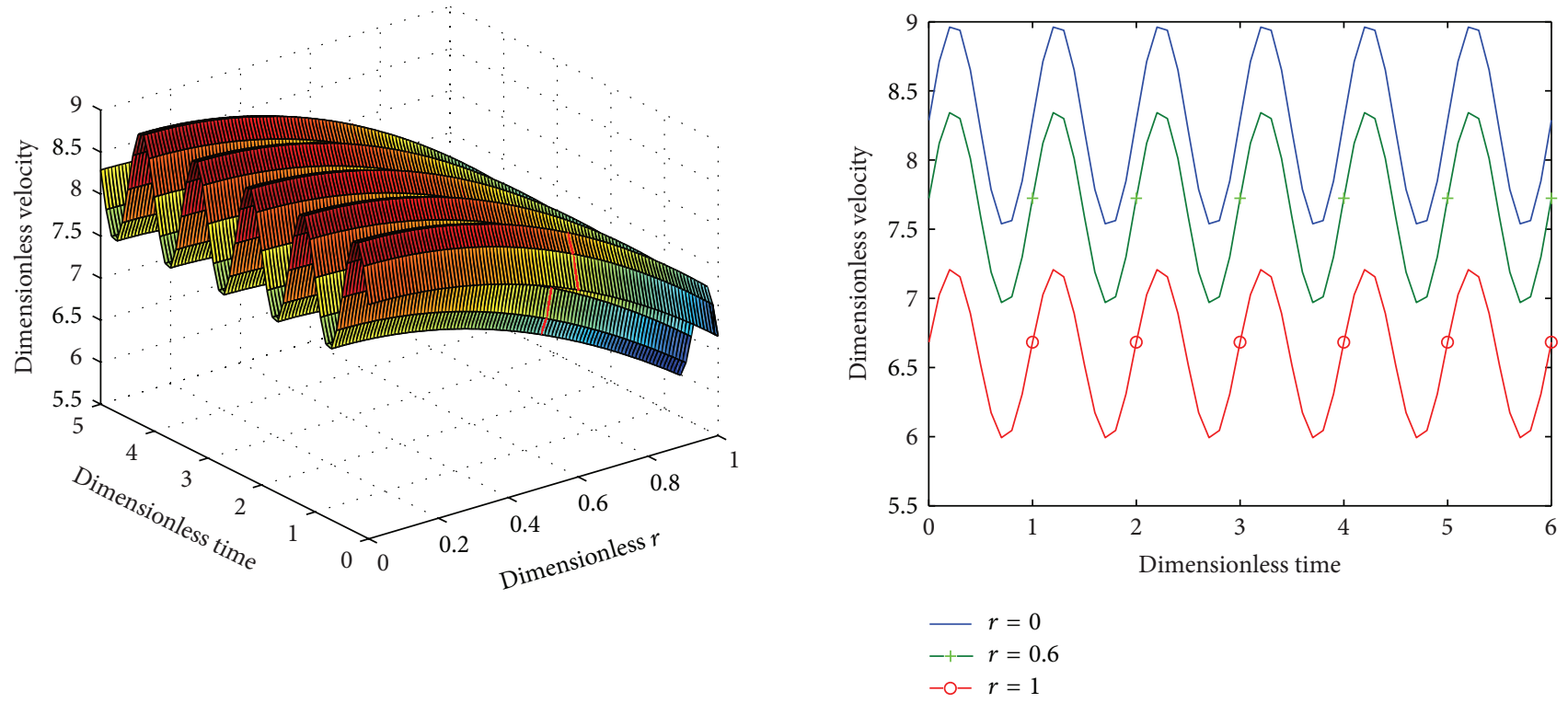

(b)

Figure 2: The velocity profile in the large artery with radius $0.50 \mathrm{~cm}$ under two different slip parameter values: (a) $l_{b}=0$; (b) $l_{b}=2$. In the figure, the 3D graphs show the variation of velocity as a function of time and location, while the 2D graphs show the variation of velocity with time at three radial locations including the artery centre $(r=0)$, the interface of inner-outer layer $(r=0.6)$, and the arterial wall $(r=1)$.

\section{Mathematical Formulation}

The flow of a fluid with no thermochemical and electromagnetic effects can be described by the conservation equations of mass and linear momentum; namely,

$$
\begin{gathered}
\frac{\partial \rho}{\partial t}+\operatorname{div}(\rho \mathbf{v})=0 \\
\rho\left(\frac{\partial \mathbf{v}}{\partial t}+\mathbf{v} \cdot \nabla \mathbf{v}\right)=\operatorname{div} \mathbf{T}+\rho \mathbf{b}
\end{gathered}
$$

where $\rho$ is the density of the fluid, $\partial / \partial t$ is the partial derivative with respect to time, $\mathbf{v}$ is the velocity vector, $\mathbf{b}$ is the body force vector, and $\mathbf{T}$ is the stress tensor.

The stress tensor is related to the velocity gradient by the constitutive equations. For a modified (generalized) secondgrade fluid [11, 31, 32], the constitutive equations can be expressed by

$$
\mathbf{T}=-p \mathbf{I}+\Pi^{m / 2}\left(\mu \mathbf{A}_{1}+\alpha_{1} \mathbf{A}_{2}+\alpha_{2} \mathbf{A}_{1}^{2},\right),
$$

where $m$ is a material parameter, $\Pi=(1 / 2) \operatorname{tr} \mathbf{A}_{1}^{2}$ is the second invariant of $\mathbf{A}_{1}, p$ is the fluid pressure, $\mu$ is the 


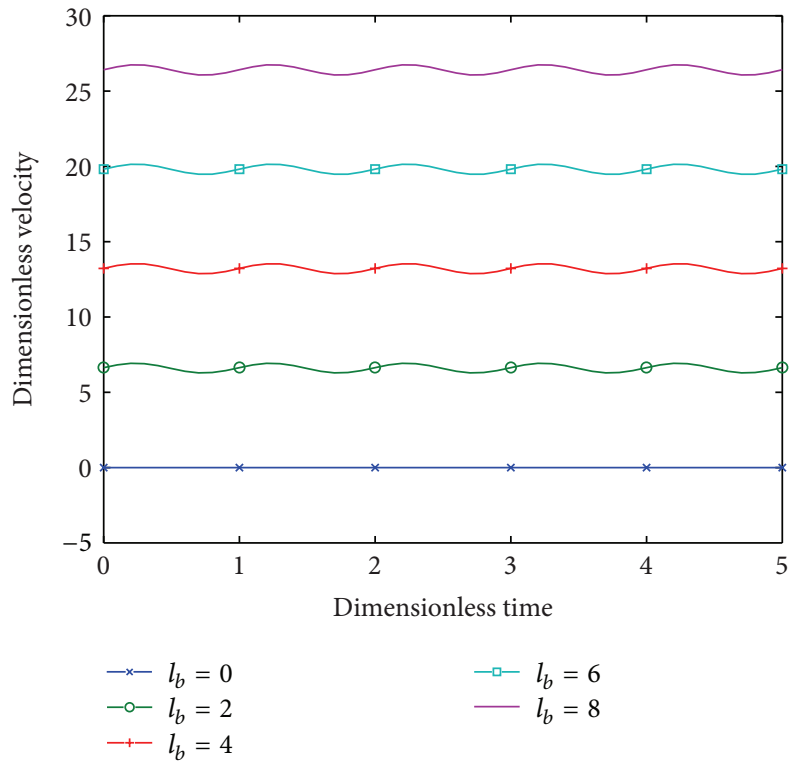

(a)

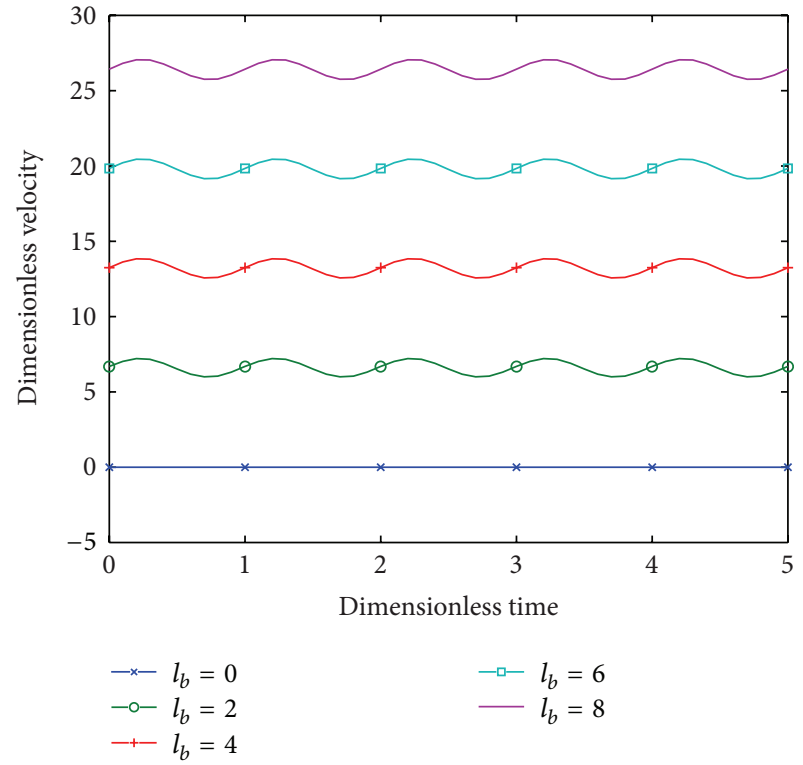

(b)

FIGURE 3: Diagrams showing the velocity profile on the arterial wall with five different slip parameters $l_{b}$ for two different artery radii (a) $r=0.15 \mathrm{~cm}$; (b) $r=0.5 \mathrm{~cm}$.

coefficient of viscosity, $\alpha_{i}$ are material moduli (the normal stress coefficients), and $\mathbf{A}_{\mathbf{i}}$ are the kinematical tensors given by

$$
\begin{gathered}
\mathbf{A}_{\mathbf{1}}=\mathbf{L}+\mathbf{L}^{T}, \\
\mathbf{A}_{\mathbf{2}}=\frac{\partial \mathbf{A}_{\mathbf{1}}}{\partial t}+\left[\operatorname{grad}\left(\mathbf{A}_{\mathbf{1}}\right)\right] \mathbf{v}+\mathbf{A}_{\mathbf{1}} \mathbf{L}+(\mathbf{L})^{T} \mathbf{A}_{\mathbf{1}},
\end{gathered}
$$

in which $L$ is $\operatorname{grad} \mathbf{v}$ and the superscript $T$ refers to matrix transposition.

For the axially symmetrical blood flow through a circular tube of radius $b$, we can assume that $\mathbf{v}=v(r, t) \mathbf{e}_{\mathrm{z}}$, where $z$ is the axial direction and $r$ is the radial direction. Under the periodic body acceleration and a unsteady pulsatile pressure gradient $[7,10]$, the momentum equation in the $z$-direction in the cylindrical polar coordinate $(r, \theta, z)$ is

$$
\rho \frac{\partial v}{\partial t}=-\frac{\partial p}{\partial z}+\rho G+\frac{1}{r} \frac{\partial}{\partial r}\left(r T_{r z}\right) .
$$

The shear stress $T_{r z}$ for a generalized second-grade fluid can be expressed by

$$
T_{r z}= \begin{cases}\mu_{1}\left|\frac{\partial v_{1}}{\partial r}\right|^{m} \frac{\partial v_{1}}{\partial r} & 0 \leq r \leq a, \\ \mu_{2} \frac{\partial v_{2}}{\partial r} & a \leq r \leq b .\end{cases}
$$

The approximate periodic form of the pressure gradient generated by the heart can be described by

$$
-\frac{\partial p}{\partial z}=A_{0}+A_{1} \cos \omega_{p} t
$$

where $A_{0}, A_{1}, \omega_{p}=2 \pi f_{p}$, and $f_{p}$ are the constant component of the pressure gradient, the amplitude of the pressure fluctuation (establishing the systolic and diastolic pressures), the circular frequency, and the frequency of pulse rate, respectively.

The body acceleration $G$ can be approximated by

$$
G=A_{g} \cos \left(\omega_{b} t+\phi\right)
$$

where $A_{g}$ is the amplitude, $f_{b}=\omega_{b} / 2 \pi$ is the frequency, and $\phi$ is the lead angle of $G$ with respect to the action of the heart.

Substituting (5)-(7) into (4), the blood flow equation for a modified second-grade fluid in the $z$-direction, in the inner and outer core, becomes

$$
\begin{aligned}
\rho_{1} \frac{\partial v_{1}}{\partial t}= & A_{0}+A_{1} \cos \omega_{p} t+\rho A_{g} \cos \left(\omega_{b} t+\phi\right) \\
& +\frac{1}{r} \frac{\partial}{\partial r}\left(r \mu_{1}\left|\frac{\partial v_{1}}{\partial r}\right|^{m} \frac{\partial v_{1}}{\partial r}\right), \quad \text { for } 0 \leq r \leq a, \\
\rho_{2} \frac{\partial v_{2}}{\partial t}= & A_{0}+A_{1} \cos \omega_{p} t+\rho A_{g} \cos \left(\omega_{b} t+\phi\right) \\
& +\frac{1}{r} \frac{\partial}{\partial r}\left(r \mu_{2} \frac{\partial v_{2}}{\partial r}\right), \quad \text { for } a \leq r \leq b .
\end{aligned}
$$

In order to completely define the problem, boundary and initial conditions are required. In this work, the Navier slip condition is applied. That is, on the solid-fluid interface $r=b$, the axial fluid velocity, relative to the solid surface, is proportional to the shear stress on the interface. As the fluid layer near the wall is modeled as a Newtonian fluid in our model, the shear stress on the boundary is related to the shear 

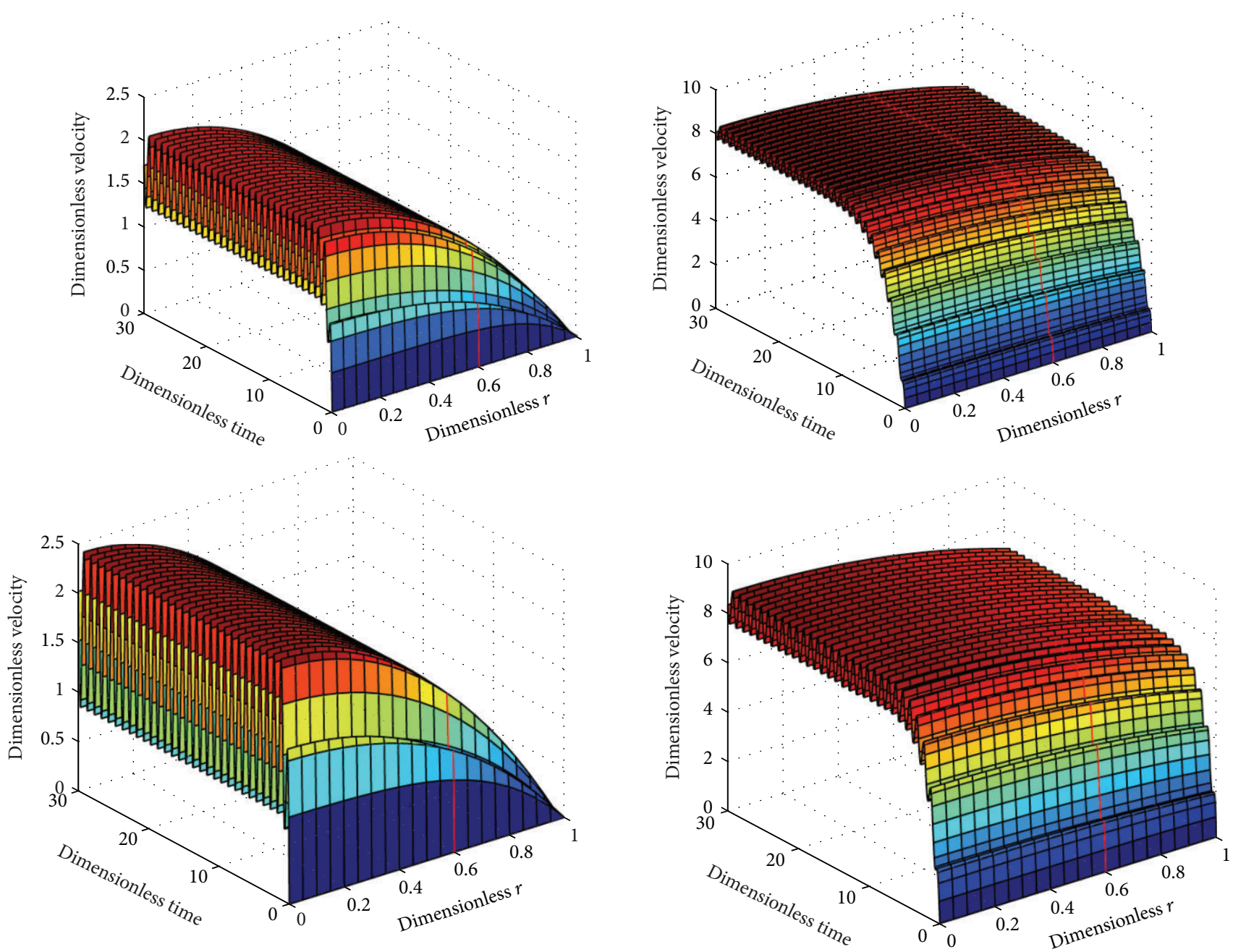

(a)

FIGURE 4: Velocity profiles in arteries with different radii $r$ : (a) $r=0.15 \mathrm{~cm}$; (b) $r=0.5 \mathrm{~cm}$. In the figure, the graphs on the left column correspond to $l_{b}=0$, while the graphs on the right column correspond to $l_{b}=2$.
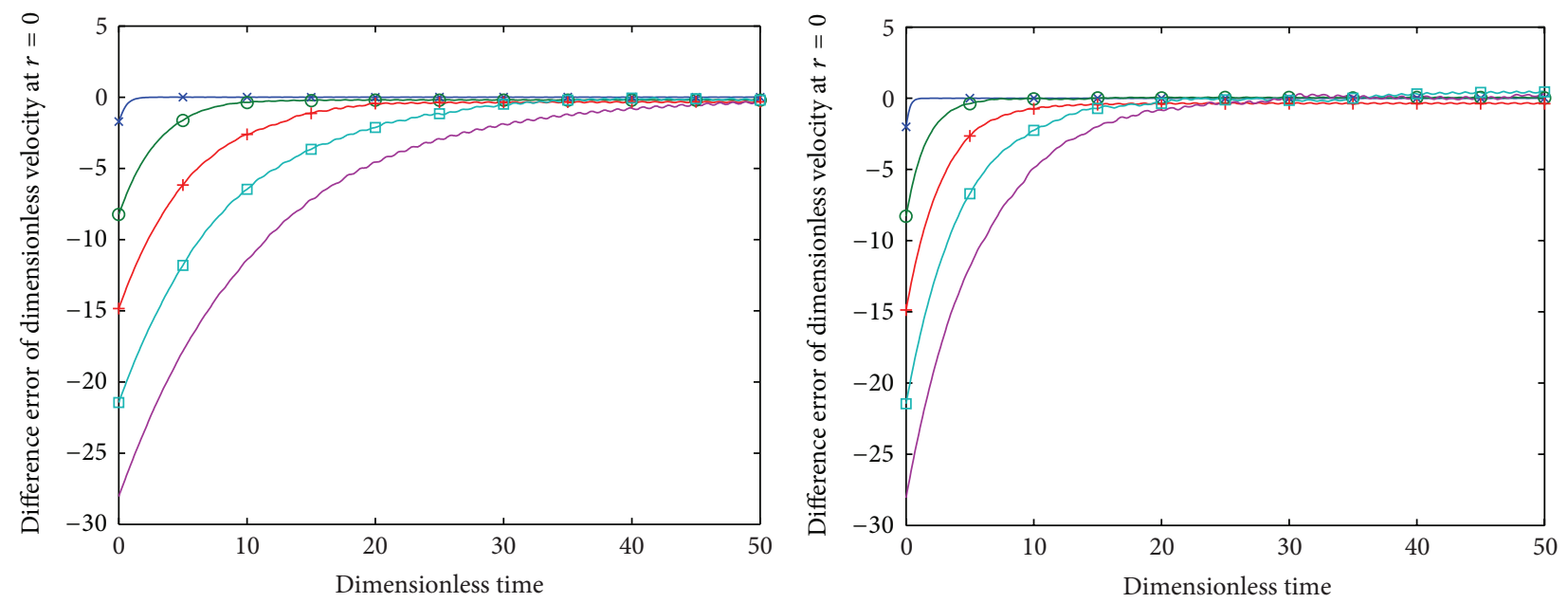
$-x-l_{b}=0$
$-\circ-l_{b}=2$
$-+-l_{b}=4$

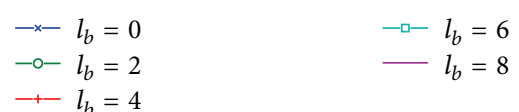

(b)

FIGURE 5: Diagrams showing the convergence of numerical solutions for different slip parameters and artery radii: (a) $r=0.15 \mathrm{~cm}$; (b) $r=0.50 \mathrm{~cm}$ 

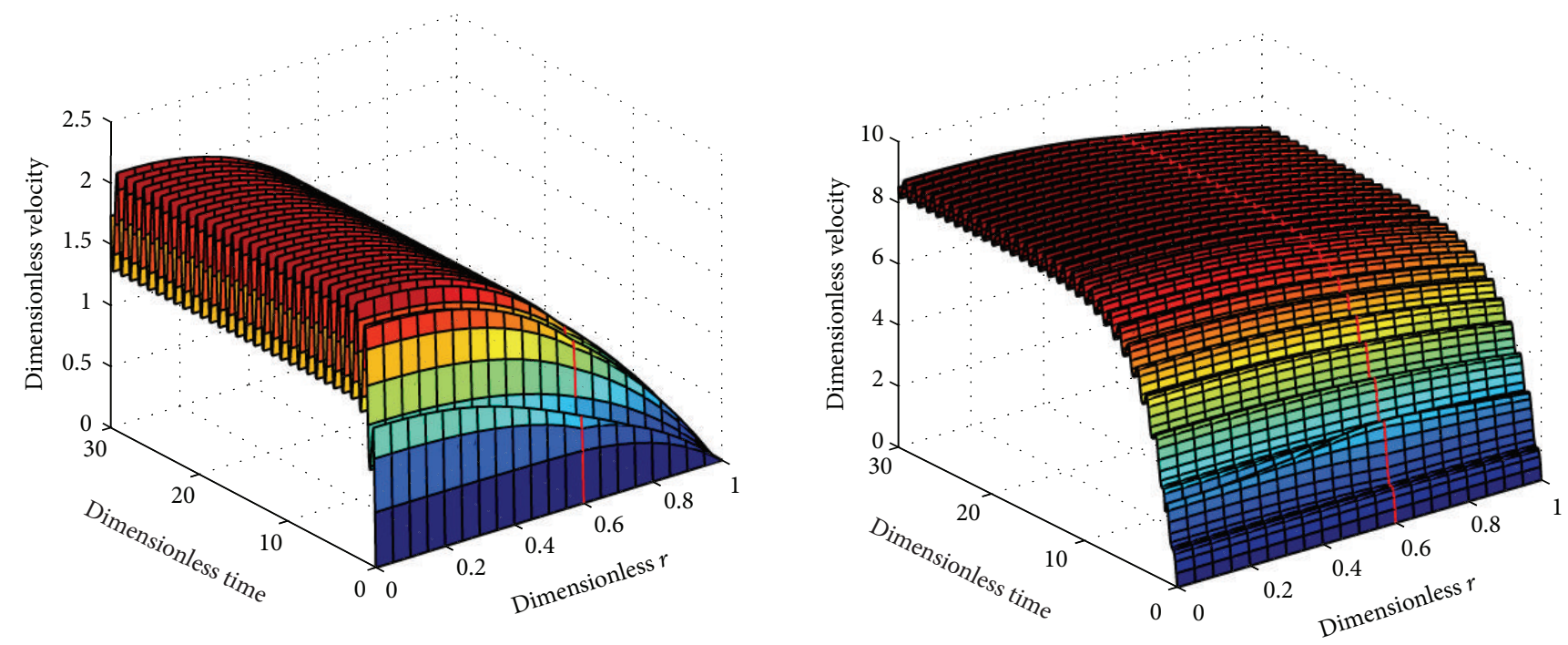

(a)
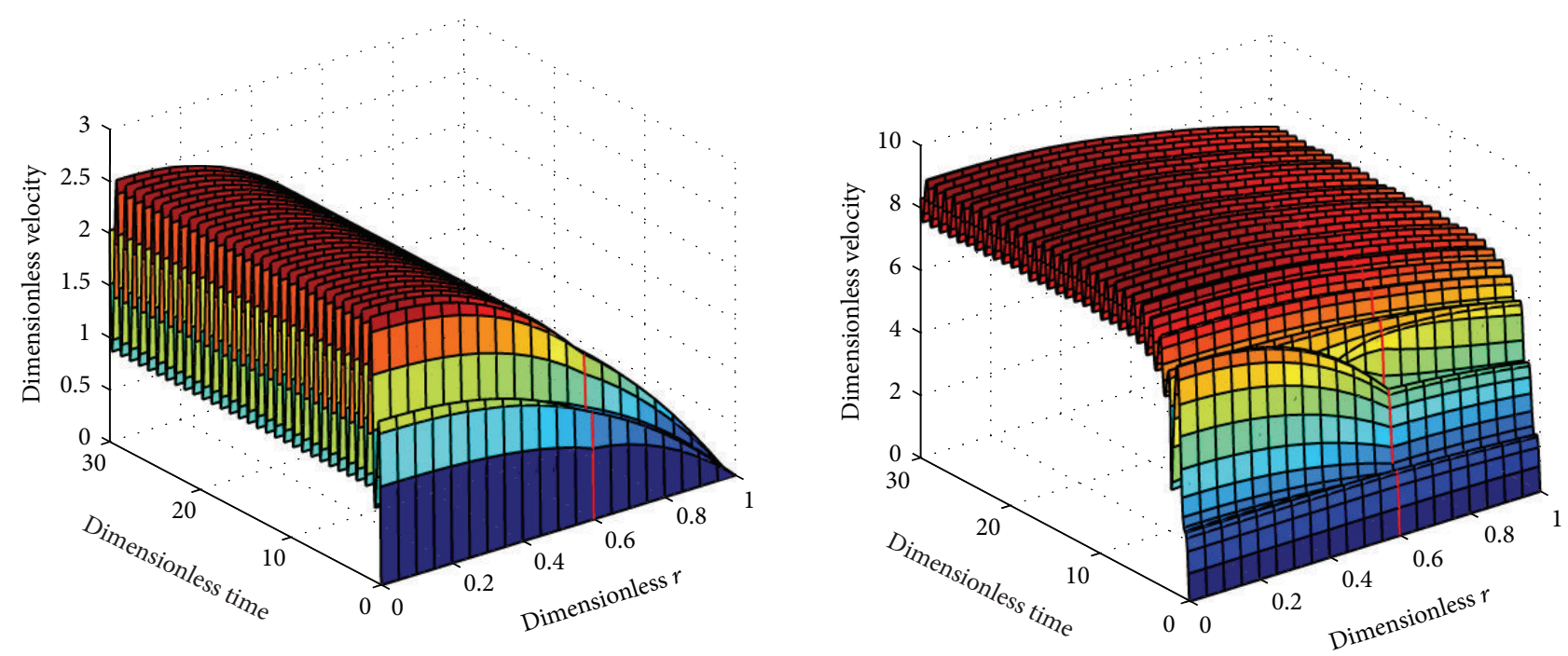

(b)

FIGURE 6: Velocity profiles in arteries with different slip parameters $l_{b}$ and radii $r$ : (a) $r=0.15 \mathrm{~cm}$; (b) $r=0.50 \mathrm{~cm}$. In the Figure, the graphs on the left column correspond to $l_{b}=0$, while the graphs on the right column correspond to $l_{b}=2$.

strain rate by $\sigma_{r z}=\mu_{2}(\partial v / \partial z)$. Thus, the Navier slip condition can be written as

$$
v_{2}(b, t)+l \frac{\partial v_{2}}{\partial t}(b, t)=0
$$

where $l$ is the slip parameter. Moreover, we assume that the slip parameter does not change along the axial direction.

On $r=0$, the symmetry condition is introduced:

$$
\frac{\partial v_{1}}{\partial r}(0, t)=0 .
$$

On the interface between two different fluids, for continuous and smooth behavior of the velocity and shear stresses, we require

$$
\begin{gathered}
v_{1}(a, t)=v_{2}(a, t), \\
{\left[\mu_{1}\left|\frac{\partial v_{1}}{\partial r}\right|^{m} \frac{\partial v_{1}}{\partial r}\right](a, t)=\left[\mu_{2} \frac{\partial v_{2}}{\partial r}\right](a, t) .}
\end{gathered}
$$

The initial conditions are set to

$$
v_{1}(r, 0)=0=v_{2}(r, 0),
$$



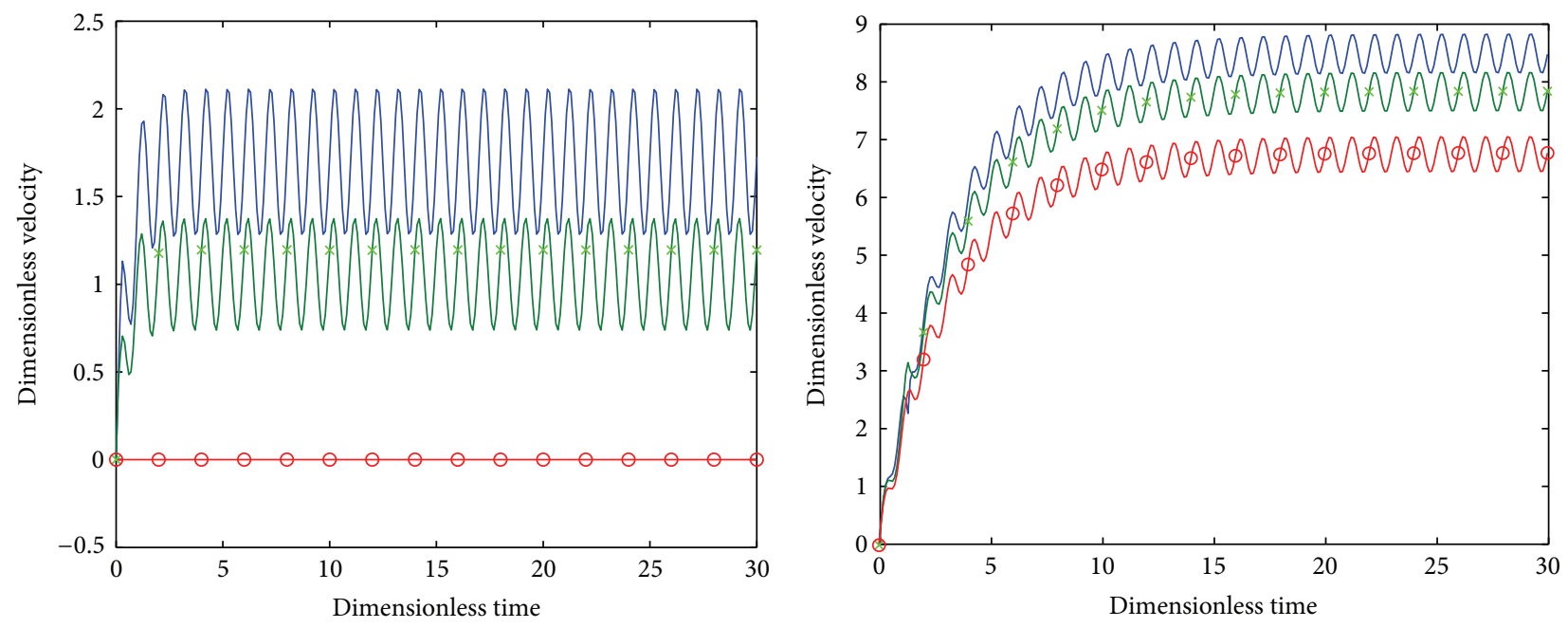

(a)
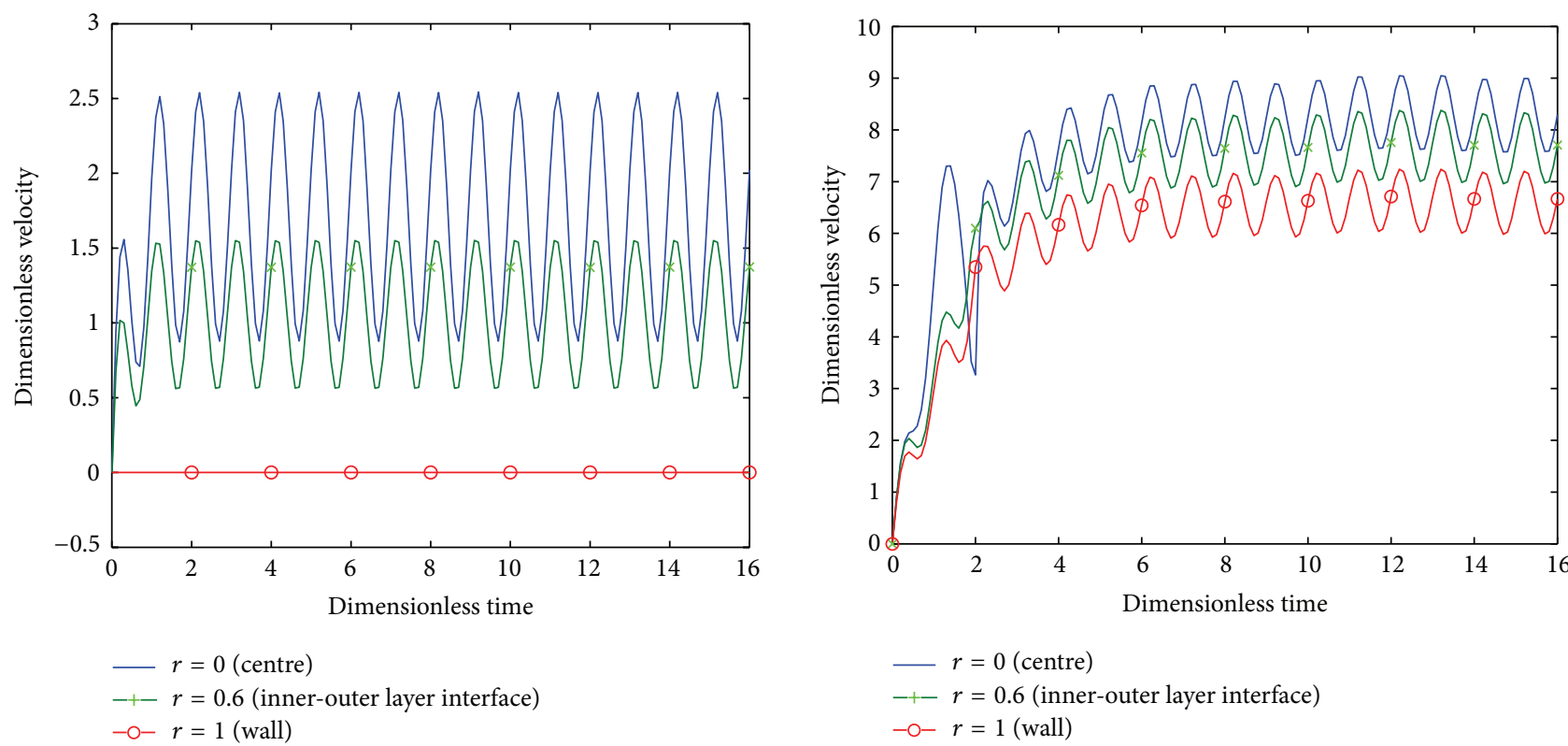

(b)

Figure 7: Velocity profiles at three arterial locations $\left(r_{1}, r_{2}, r_{3}\right)$ : for $m=-1 / 4$ and under different slip parameters $l_{b}$ and artery radii (a) $r=0.15 \mathrm{~cm}$; (b) $r=0.50 \mathrm{~cm}$. In the Figure, the graphs on the left column correspond to $l_{b}=0$, while the graphs on the right column correspond to $l_{b}=2$.

which is essential for the numerical scheme adopted to estimate the time at which the pulsatile steady state is achieved.

To simplify the equations, we introduce the following nondimensional variables and parameters:

$$
\begin{array}{cccc}
\bar{r}=\frac{r}{b}, & \bar{v}=\frac{v}{v_{0}}, & \bar{t}=\frac{\omega_{p}}{2 \pi} t, & u_{0}=\frac{A_{0} b^{2}}{\mu_{2}}, \\
e=\frac{A_{1}}{A_{0}}, & \omega_{r}=\frac{\omega_{b}}{\omega_{p}}, & r_{0}=\frac{a}{b}, & \overline{m u}=\mu\left(\frac{u_{0}}{b}\right)^{m},
\end{array}
$$$$
\begin{gathered}
\rho^{*}=\frac{\rho_{1}}{\rho_{2}}, \quad \mu^{*}=\frac{\mu_{2}}{\bar{\mu}}, \\
C_{1}=\frac{A_{0} b^{2}}{\bar{\mu} u_{0}}, \quad C_{2}=\rho_{1} A_{g} \frac{b^{2}}{\bar{\mu} u_{0}}=\frac{\rho_{1} A_{g}}{A_{0}} B_{1}, \\
\alpha=\frac{\rho_{1} \omega_{p} b^{2}}{2 \pi \bar{\mu}}, \quad \gamma=\frac{\rho_{2} \omega_{p} b^{2}}{2 \pi \bar{\mu} \mu^{*}}=\frac{\rho_{2} \omega_{p} b^{2} \rho_{1}}{2 \pi \bar{\mu} \mu^{*} \rho_{1}}=\alpha \frac{\rho^{*}}{\mu^{*}}, \\
\widehat{C}_{1}=\frac{A_{0} b^{2}}{\bar{\mu} u_{0} \mu^{*}}=\frac{C_{1}}{\mu^{*}}, \quad \widehat{C}_{2}=\frac{\rho_{2} A_{g} b^{2} \rho_{1}}{\bar{\mu} u_{0} \mu^{*} \rho_{1}}=C_{2} \frac{\rho^{*}}{\mu^{*}} .
\end{gathered}
$$ 


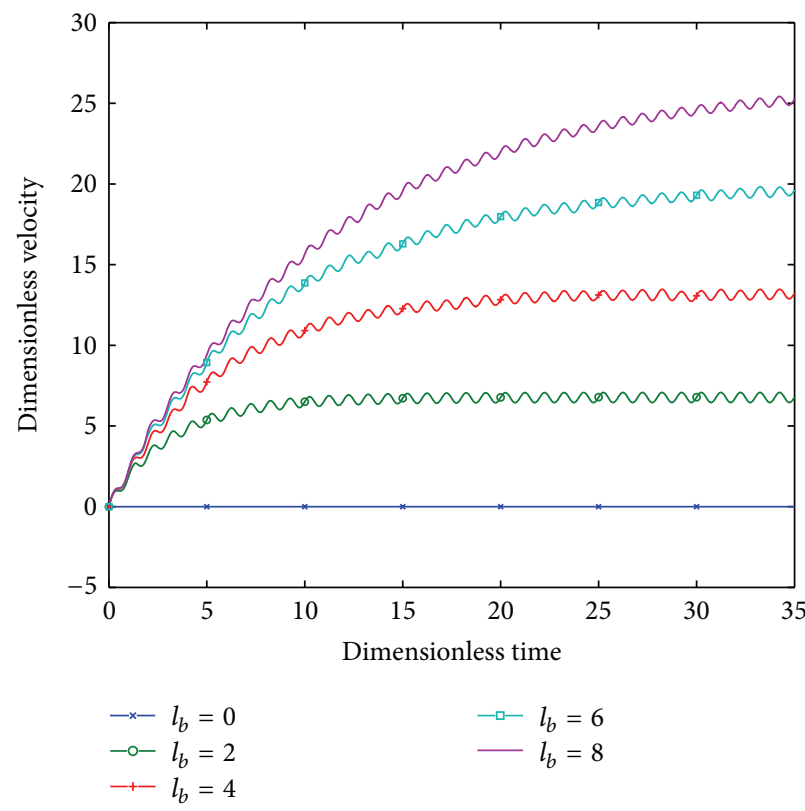

(a)

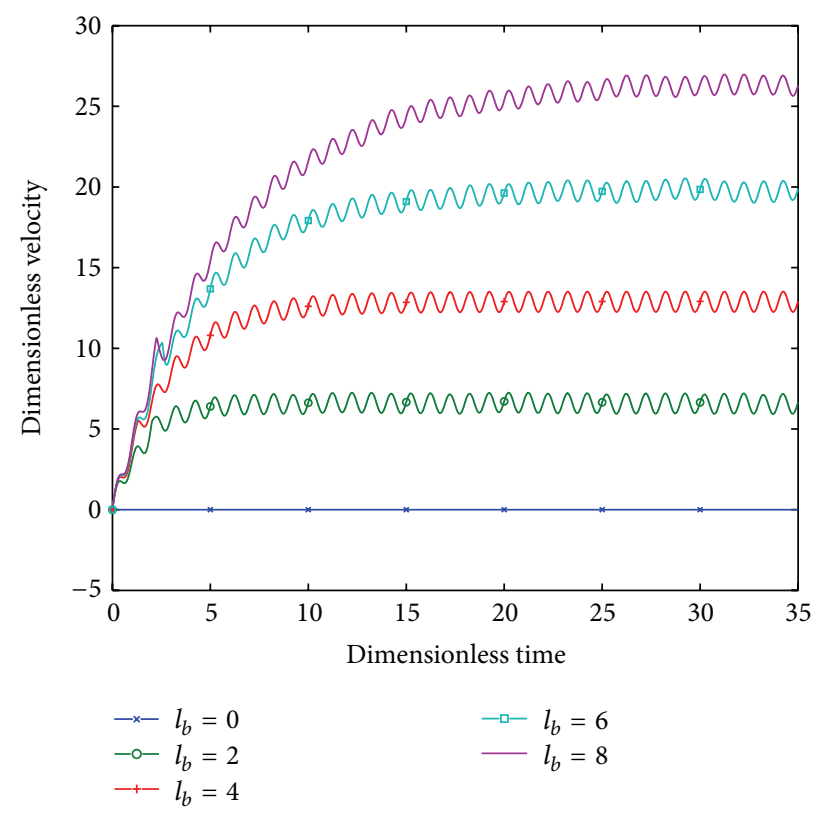

(b)

FIGURE 8: Diagrams showing the convergence of numerical results of the fluid velocity on the wall to the steady state pulsatile velocity field under various slip parameters $l_{b}$ for two different artery radii: (a) $r=0.15 \mathrm{~cm}$; (b) $r=0.50 \mathrm{~cm}$.

In terms of the nondimensional variables and parameters, (8)-(12) can be written in the form of

$$
\begin{aligned}
\alpha \frac{\partial \bar{v}_{1}}{\partial \bar{t}}= & C_{1}(1+e \cos 2 \pi \bar{t})+C_{2} \cos \left(2 \pi \omega_{r} \bar{t}+\phi\right) \\
& +\frac{1}{\bar{r}} \frac{\partial}{\partial \bar{r}}\left[\bar{r}\left|\frac{\partial \bar{v}_{1}}{\partial \bar{r}}\right|^{m} \frac{\partial \bar{v}_{1}}{\partial \bar{r}}\right], \quad \text { for } 0 \leq r \leq r_{0} \\
\gamma \frac{\partial \bar{v}_{2}}{\partial \bar{t}}= & \bar{C}_{1}(1+e \cos 2 \pi \bar{t})+\bar{C}_{2} \cos \left(2 \pi \omega_{r} \bar{t}+\phi\right) \\
& +\frac{1}{\bar{r}} \frac{\partial}{\partial \bar{r}}\left[\bar{r} \frac{\partial \bar{v}_{2}}{\partial \bar{r}}\right], \quad \text { for } r_{0} \leq r \leq 1 .
\end{aligned}
$$

The boundary conditions and initial conditions, in dimensionless form, can be expressed by

$$
\begin{gathered}
\frac{\partial \bar{v}_{1}}{\partial \bar{r}}(0, \bar{t})=0 \\
b \bar{v}_{2}(1, t)+l \frac{\partial \bar{v}_{2}}{\partial \bar{r}}(1, \bar{t})=0, \\
\bar{v}_{1}\left(r_{0}, \bar{t}\right)=\bar{v}_{2}\left(r_{0}, \bar{t}\right), \\
{\left[\left|\frac{\partial \bar{v}_{1}}{\partial \bar{r}}\right|^{m} \frac{\partial \bar{v}_{1}}{\partial \bar{r}}\right]\left(r_{0}, \bar{t}\right)=\left[\mu^{*} \frac{\partial \bar{v}_{2}}{\partial \bar{r}}\right]\left(r_{0}, \bar{t}\right),} \\
\bar{v}_{1}(r, 0)=0=\bar{v}_{2}(r, 0) .
\end{gathered}
$$

\section{Analytical Solution}

For $m=0$, the model reduces to the linear model with different viscosity in the peripheral layer and the centre core. In this case, (14) have the same form:

$$
\begin{aligned}
L(v) & =\beta \frac{\partial v}{\partial t}-\frac{1}{r} \frac{\partial v}{\partial r}-\frac{\partial^{2} v}{\partial r^{2}} \\
& =B_{1}(1+e \cos (2 \pi t))+B_{2} \cos \left(2 \pi \omega_{r} t+\phi\right) .
\end{aligned}
$$

By the superposition principle, if $v_{0}, v_{1}$, and $v_{2}$ are the solution of $L(v)=f(t)$, respectively, for $f(t)=$ $B_{1} e^{0 t i}, B_{1} a e^{2 \pi t i}$, and $B_{2} e^{\left(2 \pi \omega_{r} t+\phi\right) i}$, then the complete solution of (20) is $v=\sum_{n=0}^{2} \operatorname{Re}\left(v_{n}\right)$.

To determine $v_{n}$, we solve

$$
\beta \frac{\partial v_{n}}{\partial t}=D_{n} e^{g_{n}(t) i}+\frac{1}{r} \frac{\partial v_{n}}{\partial r}+\frac{\partial^{2} v_{n}}{\partial r^{2}},
$$

where $g_{0}(t)=0, g_{1}(t)=2 \pi t, g_{2}(t)=2 \pi \omega_{r} t+\phi, D_{0}=B_{1}$, $D_{1}=a B_{1}$, and $D_{2}=B_{2}$. As (21) admits solutions of the form $v_{n}=f_{n}(r) e^{g_{n}(t) i}$, we have from (21) that

$$
\begin{aligned}
\beta g_{n}^{\prime}( & t) f_{n}(r) e^{g_{n}(t) i} i \\
= & D_{n} e^{g_{n}(t) i}+\frac{1}{r} f_{n}^{\prime}(r) e^{g_{n}(t) i}+f_{n}^{\prime \prime}(r) e^{g_{n}(t) i} .
\end{aligned}
$$

Dividing by $e^{g_{n}(t) i}$ on both sides of (22), we obtain

$$
\beta g_{n}^{\prime}(t) f_{n}(r) i=D_{n}+\frac{1}{r} f_{n}^{\prime}(r)+f_{n}^{\prime \prime}(r) .
$$


For $n=0$, we get

$$
f_{0}^{\prime \prime}(r)+\frac{1}{r} f_{0}^{\prime}(r)=-B_{1}
$$

which has the general solution: $f_{0}(r)=\left(c_{1}+c_{2} \ln r\right)-\left(B_{1} / 4\right) r^{2}$.

For $n=1$, we have

$$
f_{1}^{\prime \prime}(r)+\frac{1}{r} f_{1}^{\prime}(r)-2 \pi \beta i f_{1}(r)=-e B_{1} .
$$

Let $\bar{\beta}_{1}^{2}=-2 \pi \beta i$; then,

$$
\frac{1}{\bar{\beta}_{1}^{2}} f_{1}^{\prime \prime}(r)+\frac{1}{\bar{\beta}_{1}^{2} r} f_{1}^{\prime}(r)+f_{1}(r)=-\frac{e B_{1}}{\bar{\beta}_{1}^{2}} .
$$

Let $\widehat{r}=\bar{\beta}_{1} r$; we have

$$
\widehat{r}^{2} f_{1}^{\prime \prime}(\widehat{r})+\widehat{r} f_{1}^{\prime}(\widehat{r})+\widehat{r}^{2} f_{1}(\widehat{r})=-\frac{e B_{1}}{\bar{\beta}_{1}^{2}} \widehat{r}^{2} .
$$

The general solution of (27) is

$$
f_{1}(r)=d_{1} J_{0}\left(\bar{\beta}_{1} r\right)+e_{1} Y_{0}\left(\bar{\beta}_{1} r\right)-\frac{e B_{1}}{2 \pi \beta} i
$$

where $d_{1}$ and $e_{1}$ are integration constants and $J_{0}$ and $Y_{0}$ denote the zero-order Bessel functions of the first kind and the second kind, respectively.

Similarly, for $n=2$, we have

$$
f_{2}^{\prime \prime}(r)+\frac{1}{r} f_{2}^{\prime}(r)-2 \beta \pi \omega_{r} f_{2}(r) i=-B_{2},
$$

and the general solution is

$$
f_{2}=d_{2} J_{0}\left(\bar{\beta}_{2} r\right)+e_{2} Y_{0}\left(\bar{\beta}_{2} r\right)-\frac{B_{2}}{2 \beta \omega_{r} \pi} i,
$$

where $\bar{\beta}_{2}^{2}=-2 \pi \beta \omega_{r} i$.

Because the boundness of $v_{1}, v_{2}, c_{2}, e_{1}$, and $e_{2}$ are set to zero, hence, from (14) and the solutions for (20), we have

$$
\begin{aligned}
& \bar{v}_{1}=\operatorname{Re}\{ c_{1}-\frac{C_{1}}{4} \bar{r}^{2}+\left[d_{1} J_{0}\left(\beta_{1} \bar{r}\right)-\frac{e C_{1}}{2 \pi \alpha} i\right] e^{2 \pi \bar{t} i} \\
&+ {\left.\left[d_{2} J_{0}\left(\beta_{2} \bar{r}\right)-\frac{C_{2}}{2 \pi \omega_{r} \alpha} i\right] e^{\left(2 \pi \omega_{r} \bar{t}+\phi\right) i}\right\}, } \\
& \bar{v}_{2}=\operatorname{Re}\left\{\widehat{c}_{1}+\widehat{c}_{2} \ln \bar{r}-\frac{\bar{C}_{1}}{4} \bar{r}^{2}\right. \\
&+\left[\widehat{d}_{1} J_{0}\left(\widehat{\beta}_{1} \bar{r}\right)+\widehat{e}_{1} Y_{0}\left(\widehat{\beta}_{1} \bar{r}\right)-\frac{e \widehat{C}_{1}}{2 \pi \gamma} i\right] e^{2 \pi \bar{t} i} \\
&+\left[\widehat{d}_{2} J_{0}\left(\widehat{\beta}_{2} \bar{r}\right)+\widehat{e}_{2} Y_{0}\left(\widehat{\beta}_{2} \bar{r}\right)-\frac{\bar{c}_{2} i}{2 \pi \omega_{r} \gamma}\right] \\
&\left.\times e^{\left(2 \pi \omega_{r} \bar{t}+\phi\right) i}\right\},
\end{aligned}
$$

where $\widehat{\beta}_{1}^{2}=-2 \pi \gamma i, \widehat{\beta}_{2}^{2}=-2 \pi \omega_{r} \gamma i, \beta_{1}^{2}=-2 \pi \alpha i$, and $\beta_{2}^{2}=$ $-2 \pi \omega_{r} \alpha i$.

As $d J_{0}(x) / d x=-J_{1}(x)$ and $d Y_{0}(x) / d x=-Y_{0}(x)$, we have

$$
\begin{aligned}
\frac{\partial \bar{v}_{1}}{\partial \bar{r}}=\operatorname{Re}( & -\frac{C_{1}}{2} \bar{r}-d_{1} \beta_{1} J_{1}\left(\beta_{1} \bar{r}\right) e^{2 \pi \bar{t} i} \\
& \left.-d_{2} \beta_{2} J_{1}\left(\beta_{2} \bar{r}\right) e^{\left(2 \pi \omega_{r} \bar{t}+\phi\right) i}\right), \\
\frac{\partial \bar{v}_{2}}{\partial \bar{r}}=\operatorname{Re}( & \widehat{c_{2}} \frac{1}{\bar{r}}-\frac{\bar{C}_{1}}{2} \bar{r} \\
+ & {\left[-\widehat{d}_{1} \widehat{\beta}_{1} J_{1}\left(\widehat{\beta}_{1} \bar{r}\right)-\widehat{e}_{1} \widehat{\beta}_{1} Y_{1}\left(\widehat{\beta}_{1} \bar{r}\right)\right] e^{2 \pi \bar{t} i} } \\
+ & {\left.\left[-\widehat{d}_{2} \widehat{\beta}_{2} J_{1}\left(\widehat{\beta}_{2} \bar{r}\right)-\widehat{e}_{2} \widehat{\beta}_{2} Y_{1}\left(\widehat{\beta}_{2} \bar{r}\right)\right] e^{\left(2 \pi \omega_{r} \bar{t}+\phi\right) i}\right) . }
\end{aligned}
$$

Obviously, $v_{1}$ satisfies the boundary condition (15) automatically. We now consider the boundary condition (16); namely,

$$
\begin{aligned}
& \operatorname{Re}\left[\left(b \widehat{c}_{1}+l \widehat{c}_{2}-\left(l+\frac{b}{2}\right) \frac{\bar{C}_{1}}{2}\right)\right. \\
& +\left(\left(b J_{0}\left(\widehat{\beta}_{1}\right)-l \widehat{\beta}_{1} J_{1}\left(\widehat{\beta}_{1}\right)\right) \widehat{d}_{1}\right. \\
& \left.\quad+\left(b Y_{0}\left(\widehat{\beta}_{1}\right)-l \widehat{\beta}_{1} Y_{1}\left(\widehat{\beta}_{1}\right)\right) \widehat{e}_{1}-\frac{e b \bar{C}_{1} i}{2 \pi \gamma}\right) e^{2 \pi \bar{t} i} \\
& +\left(\widehat{d}_{2}\left(b J_{0}\left(\widehat{\beta}_{2}\right)-l \widehat{\beta}_{2} J_{1}\left(\widehat{\beta}_{2}\right)\right)\right. \\
& +\widehat{e}_{2}\left(b Y_{0}\left(\widehat{\beta}_{2}\right)-l \widehat{\beta}_{2} Y_{1}\left(\widehat{\beta}_{2}\right)\right) \\
& \left.\left.\quad-\frac{b \bar{C}_{2}}{2 \pi \omega_{r} \gamma} i\right) e^{\left(2 \pi \omega_{r} \bar{t}+\phi\right) i}\right]=0 .
\end{aligned}
$$

Further, from boundary conditions (17) and (18), we have

$$
\begin{aligned}
& \operatorname{Re}\left[\left(c_{1}-\widehat{c}_{1}-\widehat{c}_{2} \ln r_{0}-\left(C_{1}-\widehat{C}_{1}\right) \frac{r_{0}^{2}}{4}\right)\right. \\
& +\left(d_{1} J_{0}\left(\beta_{1} r_{0}\right)-\widehat{d}_{1} J_{0}\left(\widehat{\beta}_{1} r_{0}\right)-\widehat{e}_{1} Y_{0}\left(\widehat{\beta}_{1} r_{0}\right)\right. \\
& \left.\quad-\left(\gamma C_{1}-\alpha \bar{C}_{1}\right) \frac{e i}{2 \pi \alpha \gamma}\right) e^{2 \pi \bar{t} i} \\
& +\left(d_{2} J_{0}\left(\beta_{2} r_{0}\right)-\widehat{d}_{2} J_{0}\left(\widehat{\beta}_{2} r_{0}\right)\right. \\
& \left.\quad-\widehat{e}_{2} Y_{0}\left(\widehat{\beta}_{2} r_{0}\right)-\left(\gamma C_{2}-\alpha \bar{C}_{2}\right) \frac{i}{2 \pi \omega_{r} \gamma \alpha}\right) \\
& \left.\times e^{\left(2 \pi \omega_{r} \bar{t}+\phi\right) i}\right]=0
\end{aligned}
$$




$$
\begin{aligned}
\operatorname{Re}[( & \left.\left(\mu^{*} \bar{C}_{1}-C_{1}\right) \frac{r_{0}}{2}-\mu^{*} \frac{\widehat{c}_{2}}{r_{0}}\right) \\
+ & \left(-d_{1} \beta_{1} J_{1}\left(\beta_{1} r_{0}\right)+\widehat{d}_{1} \mu^{*} \widehat{\beta}_{1} J_{1}\left(\widehat{\beta}_{1} r_{0}\right)\right. \\
& \left.+\widehat{e}_{1} \mu^{*} \widehat{\beta}_{1} Y_{1}\left(\widehat{\beta}_{1} r_{0}\right)\right) e^{2 \pi \bar{t} i} \\
+ & \left(-d_{2} \beta_{2} J_{1}\left(\beta_{2} r_{0}\right)+\widehat{d}_{2} \mu^{*} \widehat{\beta}_{2} J_{1}\left(\widehat{\beta}_{2} r_{0}\right)\right. \\
& \left.+\widehat{e}_{2} \mu^{*} \widehat{\beta}_{2} Y_{1}\left(\widehat{\beta}_{2} r_{0}\right)\right) \\
& \left.\times e^{\left(2 \pi \omega_{r} \bar{t}+\phi\right) i}\right]=0 .
\end{aligned}
$$

As (33)-(34) must be satisfied for any instant of time $t$, we require that the constant terms and the coefficients of the exponential terms all vanish; namely,

$$
\begin{aligned}
& b \widehat{c}_{1}+l \widehat{c}_{2}-\left(l+\frac{b}{2}\right) \frac{\bar{C}_{1}}{2}=0 \\
& c_{1}-\widehat{c}_{1}-\widehat{c}_{2} \ln r_{0}-\left(C_{1}-\bar{C}_{1}\right) \frac{r_{0}^{2}}{4}=0, \\
& \left(\mu^{*} \bar{C}_{1}-C_{1}\right) \frac{r_{0}}{2}-\mu^{*} \frac{\widehat{c}_{2}}{r_{0}}=0 \text {, } \\
& \widehat{d}_{1}\left(b J_{0}\left(\widehat{\beta}_{1}\right)-l \widehat{\beta}_{1} J_{1}\left(\widehat{\beta}_{1}\right)\right)+\widehat{e}_{1}\left(b Y_{0}\left(\widehat{\beta}_{1}\right)-l \widehat{\beta}_{1} Y_{1}\left(\widehat{\beta}_{1}\right)\right) \\
& -\frac{e b \bar{C}_{1}}{2 \pi \gamma} i=0, \\
& d_{1} J_{0}\left(\beta_{1} r_{0}\right)-\widehat{d}_{1} J_{0}\left(\widehat{\beta}_{1} r_{0}\right)-\widehat{e}_{1} Y_{0}\left(\widehat{\beta}_{1} r_{0}\right)-\frac{e C_{1}}{2 \pi \alpha} i \\
& +\frac{e \bar{C}_{1}}{2 \pi \gamma} i=0, \\
& -d_{1} \beta_{1} J_{1}\left(\beta_{1} r_{0}\right)+\widehat{d}_{1} \mu^{*} \widehat{\beta}_{1} J_{1}\left(\widehat{\beta}_{1} r_{0}\right)+\widehat{e}_{1} \mu^{*} \widehat{\beta}_{1} Y_{1}\left(\widehat{\beta}_{1} r_{0}\right) \\
& =0 \text {, } \\
& \widehat{d}_{2}\left(b J_{0}\left(\widehat{\beta}_{2}\right)-l \widehat{\beta}_{2} J_{1}\left(\widehat{\beta}_{2}\right)\right)+\widehat{e}_{2}\left(b Y_{0}\left(\widehat{\beta}_{2}\right)-l \widehat{\beta}_{2} Y_{1}\left(\widehat{\beta}_{2}\right)\right) \\
& -\frac{b \bar{C}_{2}}{2 \pi \omega_{r} \gamma} i=0 \\
& d_{2} J_{0}\left(\beta_{2} r_{0}\right)-\widehat{d}_{2} J_{0}\left(\widehat{\beta}_{2} r_{0}\right)-\widehat{e}_{2} Y_{0}\left(\widehat{\beta}_{2} r_{0}\right)-\frac{C_{1}}{2 \pi \omega_{r} \alpha} i \\
& +\frac{\bar{C}_{2}}{2 \pi \omega_{r} \gamma} i=0, \\
& -d_{2} \beta_{2} J_{1}\left(\beta_{2} r_{0}\right)+\widehat{d}_{2} \mu^{*} \widehat{\beta}_{2} J_{1}\left(\widehat{\beta}_{2} r_{0}\right)+\widehat{e}_{2} \mu^{*} \widehat{\beta}_{2} Y_{1}\left(\widehat{\beta}_{2} r_{0}\right) \\
& =0 \text {. }
\end{aligned}
$$

Solving the above system of equations yields

$$
\begin{aligned}
c_{1}= & \left(\ln r_{0}-\frac{l}{b}\right)\left(\left(\mu^{*} \bar{C}_{1}-C_{1}\right) \frac{r_{0}^{2}}{2 \mu^{*}}\right) \\
& +\left(\frac{l}{b}+\frac{1-r_{0}^{2}}{2}\right) \frac{\bar{C}_{1}}{2}+C_{1} \frac{r_{0}^{2}}{4}, \\
\widehat{c}_{1}= & -\frac{l}{b}\left(\left(\mu^{*} \bar{C}_{1}-C_{1}\right) \frac{r_{0}^{2}}{2 \mu^{*}}\right)+\left(\frac{l}{b}+\frac{1}{2}\right) \frac{\bar{C}_{1}}{2}, \\
\widehat{c}_{2}= & \left(\mu^{*} \bar{C}_{1}-C_{1}\right) \frac{r_{0}^{2}}{2 \mu^{*}},
\end{aligned}
$$

$d_{1}=\mu^{*}\left[\left(J_{1}\left(\widehat{\beta}_{1} r_{0}\right) Y_{0}\left(\widehat{\beta}_{1} r_{0}\right)-J_{0}\left(\widehat{\beta}_{1} r_{0}\right) Y_{1}\left(\widehat{\beta}_{1} r_{0}\right)\right)\right.$

$$
\begin{aligned}
& \times \frac{e b \widehat{\beta}_{1} \bar{C}_{1} i}{2 \pi \gamma}+\left(\gamma C_{1}-\alpha \bar{C}_{1}\right) \\
& \times \\
& \quad\left[J_{1}\left(\widehat{\beta}_{1} r_{0}\right)\left(b Y_{0}\left(\widehat{\beta}_{1}\right)-l \widehat{\beta}_{1} Y_{1}\left(\widehat{\beta}_{1}\right)\right)\right. \\
& \left.\quad-Y_{1}\left(\widehat{\beta}_{1} r_{0}\right)\left(b J_{0}\left(\widehat{\beta}_{1}\right)-l \widehat{\beta}_{1} J_{1}\left(\widehat{\beta}_{1}\right)\right)\right] \\
& \left.\times \frac{\widehat{\beta}_{1} e i}{2 \pi \gamma \alpha}\right] /\left(b J_{0}\left(\widehat{\beta}_{1}\right)-l \widehat{\beta}_{1} J_{1}\left(\widehat{\beta}_{1}\right)\right)
\end{aligned}
$$$$
\times\left(\beta_{1} J_{1}\left(\beta_{1} r_{0}\right) Y_{0}\left(\widehat{\beta}_{1} r_{0}\right)-\mu^{*} \widehat{\beta}_{1} Y_{1}\left(\widehat{\beta}_{1} r_{0}\right) J_{0}\left(\beta_{1} r_{0}\right)\right)
$$$$
+\left(b Y_{0}\left(\widehat{\beta}_{1}\right)-l \widehat{\beta}_{1} Y_{1}\left(\widehat{\beta}_{1}\right)\right)
$$$$
\times\left(\mu^{*} \widehat{\beta}_{1} J_{0}\left(\beta_{1} r_{0}\right) J_{1}\left(\widehat{\beta}_{1} r_{0}\right)-\beta_{1} J_{1}\left(\beta_{1} r_{0}\right) J_{0}\left(\widehat{\beta}_{1} r_{0}\right)\right),
$$$$
\widehat{d}_{1}=\left[\left(\beta_{1} J_{1}\left(\beta_{1} r_{0}\right) Y_{0}\left(\widehat{\beta}_{1} r_{0}\right)\right.\right.
$$

$$
\left.-\mu^{*} \widehat{\beta}_{1} Y_{1}\left(\widehat{\beta}_{1} r_{0}\right) J_{0}\left(\beta_{1} r_{0}\right)\right)
$$$$
\times \frac{e b \bar{C}_{1}}{2 \pi \gamma} i+\frac{\left(\gamma C_{1}-\alpha \bar{C}_{1}\right)}{2 \pi \gamma \alpha}
$$$$
\times\left(b Y_{0}\left(\widehat{\beta}_{1}\right)-l \widehat{\beta}_{1} Y_{1}\left(\widehat{\beta}_{1}\right)\right)
$$$$
\left.\times e \beta_{1} J_{1}\left(\beta_{1} r_{0}\right) i\right] /\left(b J_{0}\left(\widehat{\beta}_{1}\right)-l \widehat{\beta}_{1} J_{1}\left(\widehat{\beta}_{1}\right)\right)
$$$$
\times\left(\beta_{1} J_{1}\left(\beta_{1} r_{0}\right) Y_{0}\left(\widehat{\beta}_{1} r_{0}\right)\right.
$$$$
\left.-\mu^{*} \widehat{\beta}_{1} Y_{1}\left(\widehat{\beta}_{1} r_{0}\right) J_{0}\left(\beta_{1} r_{0}\right)\right)
$$$$
+\left(b Y_{0}\left(\widehat{\beta}_{1}\right)-l \widehat{\beta}_{1} Y_{1}\left(\widehat{\beta}_{1}\right)\right)
$$$$
\times\left(\mu^{*} \widehat{\beta}_{1} J_{0}\left(\beta_{1} r_{0}\right) J_{1}\left(\widehat{\beta}_{1} r_{0}\right)\right.
$$$$
\left.-\beta_{1} J_{1}\left(\beta_{1} r_{0}\right) J_{0}\left(\widehat{\beta}_{1} r_{0}\right)\right),
$$

$\widehat{e}_{1}=\left[\left(\mu^{*} \widehat{\beta}_{1} J_{0}\left(\beta_{1} r_{0}\right) J_{1}\left(\widehat{\beta}_{1} r_{0}\right)\right.\right.$

$$
\left.-\beta_{1} J_{0}\left(\widehat{\beta}_{1} r_{0}\right) J_{1}\left(\beta_{1} r_{0}\right)\right)
$$




$$
\begin{aligned}
& \times \frac{e b \bar{C}_{1} i}{2 \pi \gamma}-\left(b J_{0}\left(\widehat{\beta}_{1}\right)-l \widehat{\beta}_{1} J_{1}\left(\widehat{\beta}_{1}\right)\right) \\
& \times\left(\gamma C_{1}-\alpha \bar{C}_{1}\right) \\
& \left.\times J_{1}\left(\beta_{1} r_{0}\right) \frac{\beta_{1} e i}{2 \pi \alpha \gamma}\right] /\left(b J_{0}\left(\widehat{\beta}_{1}\right)-l \widehat{\beta}_{1} J_{1}\left(\widehat{\beta}_{1}\right)\right) \\
& \times\left(\beta_{1} J_{1}\left(\beta_{1} r_{0}\right) Y_{0}\left(\widehat{\beta}_{1} r_{0}\right)\right. \\
& \left.-\mu^{*} \widehat{\beta}_{1} Y_{1}\left(\widehat{\beta}_{1} r_{0}\right) J_{0}\left(\beta_{1} r_{0}\right)\right) \\
& +\left(b Y_{0}\left(\widehat{\beta}_{1}\right)-l \widehat{\beta}_{1} Y_{1}\left(\widehat{\beta}_{1}\right)\right) \\
& \times\left(\mu^{*} \widehat{\beta}_{1} J_{0}\left(\beta_{1} r_{0}\right) J_{1}\left(\widehat{\beta}_{1} r_{0}\right)-\beta_{1} J_{1}\left(\beta_{1} r_{0}\right) J_{0}\left(\widehat{\beta}_{1} r_{0}\right)\right), \\
& d_{2}=\mu^{*}\left[\left(J_{1}\left(\widehat{\beta}_{2} r_{0}\right) Y_{0}\left(\widehat{\beta}_{2} r_{0}\right)-J_{0}\left(\widehat{\beta}_{2} r_{0}\right) Y_{1}\left(\widehat{\beta}_{2} r_{0}\right)\right)\right. \\
& \times \frac{b \widehat{\beta}_{2} \bar{C}_{1} i}{2 \pi \omega_{r} \gamma}+\left(\gamma C_{2}-\alpha \bar{C}_{2}\right) \\
& \times\left[J_{1}\left(\widehat{\beta}_{2} r_{0}\right)\left(b Y_{0}\left(\widehat{\beta}_{2}\right)-l \widehat{\beta}_{2} Y_{1}\left(\widehat{\beta}_{2}\right)\right)\right. \\
& \left.-Y_{1}\left(\widehat{\beta}_{2} r_{0}\right)\left(b J_{0}\left(\widehat{\beta}_{2}\right)-i \widehat{\beta}_{2} J_{1}\left(\widehat{\beta}_{2}\right)\right)\right] \\
& \left.\times \frac{\widehat{\beta}_{2} i}{2 \pi \omega_{r} \gamma \alpha}\right] /\left(b J_{0}\left(\widehat{\beta}_{2}\right)-l \widehat{\beta}_{2} J_{1}\left(\widehat{\beta}_{2}\right)\right) \\
& \times\left(\beta_{2} J_{1}\left(\beta_{2} r_{0}\right) Y_{0}\left(\widehat{\beta}_{2} r_{0}\right)\right. \\
& \left.-\mu^{*} \widehat{\beta}_{2} Y_{1}\left(\widehat{\beta}_{2} r_{0}\right) J_{0}\left(\beta_{2} r_{0}\right)\right) \\
& +\left(b Y_{0}\left(\widehat{\beta}_{2}\right)-l \widehat{\beta}_{2} Y_{1}\left(\widehat{\beta}_{2}\right)\right) \\
& \times\left(\mu^{*} \widehat{\beta}_{2} J_{0}\left(\beta_{2} r_{0}\right) J_{1}\left(\widehat{\beta}_{2} r_{0}\right)\right. \\
& \left.-\beta_{2} J_{1}\left(\beta_{2} r_{0}\right) J_{0}\left(\widehat{\beta}_{2} r_{0}\right)\right), \\
& \widehat{d}_{2}=\left[\left(\beta_{2} J_{1}\left(\beta_{2} r_{0}\right) Y_{0}\left(\widehat{\beta}_{2} r_{0}\right)\right.\right. \\
& \left.-\mu^{*} \widehat{\beta}_{2} Y_{1}\left(\widehat{\beta}_{2} r_{0}\right) J_{0}\left(\beta_{2} r_{0}\right)\right) \\
& \times \frac{b \bar{C}_{1}}{2 \pi \omega_{r} \gamma} i+\frac{\left(\gamma C_{1}-\alpha \bar{C}_{1}\right)}{2 \pi \gamma \alpha \omega_{r}} \\
& \times\left(b Y_{0}\left(\widehat{\beta}_{2}\right)-l \widehat{\beta}_{2} Y_{1}\left(\widehat{\beta}_{2}\right)\right) \\
& \left.\times \beta_{2} J_{1}\left(\beta_{2} r_{0}\right) i\right] /\left(b J_{0}\left(\widehat{\beta}_{2}\right)-l \widehat{\beta}_{2} J_{1}\left(\widehat{\beta}_{2}\right)\right) \\
& \times\left(\beta_{2} J_{1}\left(\beta_{2} r_{0}\right) Y_{0}\left(\widehat{\beta}_{2} r_{0}\right)\right. \\
& \left.-\mu^{*} \widehat{\beta}_{2} Y_{1}\left(\widehat{\beta}_{2} r_{0}\right) J_{0}\left(\beta_{2} r_{0}\right)\right) \\
& +\left(b Y_{0}\left(\widehat{\beta}_{2}\right)-l \widehat{\beta}_{2} Y_{1}\left(\widehat{\beta}_{2}\right)\right) \\
& \times\left(\mu^{*} \widehat{\beta}_{2} J_{0}\left(\beta_{2} r_{0}\right) J_{1}\left(\widehat{\beta}_{2} r_{0}\right)\right. \\
& \left.-\beta_{2} J_{1}\left(\beta_{2} r_{0}\right) J_{0}\left(\widehat{\beta}_{2} r_{0}\right)\right) \text {, }
\end{aligned}
$$

$$
\begin{aligned}
\widehat{e}_{2}= & {\left[\left(\mu^{*} \widehat{\beta}_{2} J_{0}\left(\beta_{2} r_{0}\right) J_{1}\left(\widehat{\beta}_{2} r_{0}\right)\right.\right.} \\
& \left.-\beta_{2} J_{0}\left(\widehat{\beta}_{2} r_{0}\right) J_{1}\left(\beta_{2} r_{0}\right)\right) \\
\times & \frac{b \bar{C}_{1} i}{2 \pi \omega_{r} \gamma}-\left(b J_{0}\left(\widehat{\beta}_{2}\right)-l \widehat{\beta}_{2} J_{1}\left(\widehat{\beta}_{2}\right)\right) \\
\times & \left(\gamma C_{1}-\alpha \bar{C}_{1}\right) J_{1}\left(\beta_{2} r_{0}\right) \\
\times & \left.\frac{\beta_{2} i}{2 \pi \omega_{r} \alpha \gamma}\right] /\left(b J_{0}\left(\widehat{\beta}_{2}\right)-l \widehat{\beta}_{2} J_{1}\left(\widehat{\beta}_{2}\right)\right) \\
\times & \left(\beta_{2} J_{1}\left(\beta_{2} r_{0}\right) Y_{0}\left(\widehat{\beta}_{2} r_{0}\right)\right. \\
& \left.-\mu^{*} \widehat{\beta}_{2} Y_{1}\left(\widehat{\beta}_{2} r_{0}\right) J_{0}\left(\beta_{2} r_{0}\right)\right) \\
+ & \left(b Y_{0}\left(\widehat{\beta}_{2}\right)-l \widehat{\beta}_{2} Y_{1}\left(\widehat{\beta}_{2}\right)\right) \\
\times & \left(\mu^{*} \widehat{\beta}_{2} J_{0}\left(\beta_{2} r_{0}\right) J_{1}\left(\widehat{\beta}_{2} r_{0}\right)\right. \\
& \left.\quad-\beta_{2} J_{1}\left(\beta_{2} r_{0}\right) J_{0}\left(\widehat{\beta}_{2} r_{0}\right)\right) .
\end{aligned}
$$

To show the flow behavior and the effect of the slip parameter, we investigate the velocity profiles in the arteries with different values of the slip parameter under various different conditions. In the first example of investigation, the radius of the artery is taken as $r=b=0.15 \mathrm{~cm}$, and the other parameters are set to $A_{0}=698.65 \mathrm{dyne} / \mathrm{cm}^{3}, A_{g}=0.5 \mathrm{~g}$, $f_{b}=f_{p}=1.2, \phi=0, C_{1}=6.6, C_{2}=4.64, A_{1}=1.2 A_{0}$, and $\rho_{1} / \rho_{2}=1$. Figure 1 shows the 3 -dimensional velocity profile as a function of time and location and the 2-dimensional velocity profile as a function of time at three different radial locations for two different slip parameters $l=0$ (no-slip) and $l=2$. The results show that boundary slip has a very dramatical effect on the fluid flow in the artery. It affects not only the magnitude of the flow velocity significantly, but also the flow pattern and velocity profile on the cross-section of the artery. For the no-slip flow $\left(l_{b}=0\right)$, the pulsatile flow nature gradually disappears toward the arterial wall, while with boundary slip, the flow near the arterial wall also displays a pulsatile nature.

We then investigate whether the above observed flow phenomena associated with boundary slip are affected or not by the radius of the artery, and for this purpose, we consider the fluid flow through an artery with a larger radius $r=0.5 \mathrm{~cm}$. The constant pressure gradient is set to $A_{0}=32 \mathrm{dyne} / \mathrm{cm}^{3}$ in order to achieve a mean velocity magnitude approximately equal to that in the smaller artery, while all other parameters are set to the same values as those used for the smaller radius. Figure 2 shows the velocity profile in the artery for two different slip parameter values including $l_{b}=0$ (no-slip) and $l_{b}=2$. The 3 -dimensional graph shows the variation of the flow velocity with time and radial position, while the 2 -dimensional graphs demonstrate the variation of the flow velocity with time at three different radial locations including $r=0$ (centre), $r=0.6$ (inner-outer layers interface), and $r=1$ (arterial wall). From Figures 1 and 2, it is clear 
that the boundary slip related flow phenomena and behavior observed for the smaller artery also appear in the artery with a larger radius, and further, a more significant pulsatile nature of fluid flow is observed for the larger artery.

To further investigate the effect of the slip parameter on the velocity profile near the artery wall, we show in Figure 3 the velocity of fluid on the artery wall for four different values of the slip parameter including $l_{b}=0,2,4,6$, and 8 . The results clearly demonstrate that the slip parameter has a very significant effect on the near-wall velocity and that the magnitude of the average wall velocity is proportional to the slip parameter.

\section{Numerical Investigation}

A numerical scheme, based on the finite different method, is established to solve the underlying boundary value problem for the general case $m \neq 0$, consisting of (14) and boundary condition (15)-(19). To validate the numerical technique, we apply the numerical scheme to generate a series of numerical solutions for the case $m=0$ and then compare the numerical results with the exact solution derived in Section 3.

Figure 4 presents the velocity profile in the small and large arteries for two different slip parameters $l_{b}=0$ (no-slip) and $l_{b}=2$ obtained by the numerical technique. The numerical errors between the exact solution and the numerical solution, $E_{r}=V-U$, are presented in Figure 5 in which $V$ is the exact solution and $U$ is the numerical solution. The results clearly indicate that the numerical solution converges to the exact solution. This shows that a larger slip length has a lower convergence rate.

We then investigate the flow phenomena for the general case $m \neq 0$, and here we consider $m=-1 / 4$ in the investigation. Figure 6 gives the 3D graph showing the convergence of the transient velocity field to a steady state pulsatile velocity field and also demonstrating the substantial influence of boundary slip on the steady state velocity profile in both magnitude and flow pattern. Figure 7 shows the variations of velocities with time at three arterial locations for different slip parameters and artery radii and also clearly demonstrates the significant effect of boundary slip on the flow through the artery. Figure 8 shows the variation of fluid velocity along the artery wall under different slip parameters and artery radii. The results show that as the slip parameter increases, the time required for achieving convergence results increases, and the magnitude of the average steady state velocity also increases.

\section{Conclusion}

In this paper, a mathematical model for the transient pulsatile flow of fluids through vessels, taking into account boundary slip and the Fahraeus-Lindqvist effect, is established. For a special case of the underlying boundary value problem, an exact solution for the velocity field has been derived in explicit form, which provides one with an exact analytical method for investigating the flow phenomena under the special case and also a mean for validating the subsequently developed numerical scheme for generating numerical results for the general case. Our analytical and numerical studies show that for the flow of fluids with the Fahraeus-Lindqvist effect, boundary slip has a very significant influence on the magnitude of the mean flow velocity and on the flow pattern and velocity profile on the cross-section. With boundary slip, the boundary layer near the wall also displays significant pulsatile flow nature. The results also show that as the boundary slip length increases, the convergence rate of numerical results to the exact solutions decreases and the time required to achieve the steady state pulsatile flow increases.

\section{References}

[1] A. C. Eringen, "Continuum theory of dense rigid suspensions," Rheologica Acta, vol. 30, no. 1, pp. 23-32, 1991.

[2] A. C. Eringen, "A continuum theory of dense suspensions," Zeitschrift für Angewandte Mathematik und Physik, vol. 56, no. 3, pp. 529-547, 2005.

[3] T. Ariman, M. A. Turk, and N. D. Sylvester, "Microcontinuum fluid mechanics-a review," International Journal of Engineering Science, vol. 11, no. 8, pp. 905-930, 1973.

[4] M. A. Turk, N. D. Sylvester, and T. Ariman, "On pulsatile blood flow," Transactions of the Society of Rheology, vol. 17, pp. 1-21, 1973.

[5] L. Debnath, "On a microcontinuum model of pulsatile blood flow," Acta Mechanica, vol. 24, no. 3-4, pp. 165-177, 1976.

[6] G. Ahmadi, "A continuum theory of blood flow," Scientia Sinica, vol. 24, no. 10, pp. 1465-1474, 1981.

[7] S. N. Majhi and L. Usha, "Modelling the Fahraeus-Lindqvist effect through fluids of differential type," International Journal of Engineering Science, vol. 26, no. 5, pp. 503-508, 1988.

[8] K. Haldar and H. I. Andersson, "Two-layered model of blood flow through stenosed arteries," Acta Mechanica, vol. 117, pp. 221-228, 1996.

[9] D. S. Sankar and Y. Yatim, "Comparative analysis of mathematical models for blood flow in tapered constricted arteries," Abstract and Applied Analysis, vol. 2012, Article ID 235960, 34 pages, 2012.

[10] S. N. Majhi and V. R. Nair, "Pulsatile flow of third grade fluids under body acceleration-Modelling blood flow," International Journal of Engineering Science, vol. 32, no. 5, pp. 839-846, 1994.

[11] M. Massoudi and T. X. Phuoc, "Pulsatile flow of blood using a modified second-grade fluid model," Computers \& Mathematics with Applications, vol. 56, no. 1, pp. 199-211, 2008.

[12] J. C. Slattery, Advanced Transport Phenomena, Cambridge University Press, 1999.

[13] R. Pit, H. Hervet, and L. Léger, "Direct experimental evidence of slip in hexadecane: solid interfaces," Physical Review Letters, vol. 85 , no. 5, pp. 980-983, 2000.

[14] R. Tuinier and T. Taniguchi, "Polymer depletion-induced slip near an interface," Journal of Physics Condensed Matter, vol. 17, no. 2, pp. L9-L14, 2005.

[15] B.-Y. Cao, M. Chen, and Z.-Y. Guo, "Velocity slip of liquid flow in nanochannels," Acta Physica Sinica, vol. 55, no. 10, pp. 53055310, 2006.

[16] L. Szalmas, "Slip-flow boundary condition for straight walls in thelattice Boltzmann model," Physical Review E, vol. 7306, no. 6, article 6710, 2006.

[17] J. P. Pascal, "Instability of power-law fluid flow down a porous incline," Journal of Non-Newtonian Fluid Mechanics, vol. 133, no. 2-3, pp. 109-120, 2006. 
[18] K. C. Sahu, P. Valluri, P. D. M. Spelt, and O. K. Matar, "Linear instability of pressure-driven channel flow of a Newtonian and a Herschel-Bulkley fluid," Physics of Fluids, vol. 20, no. 10, Article ID 109902, 2008.

[19] J. Xu and Y. Li, "Boundary conditions at the solid-liquid surface over the multiscale channel size from nanometer to micron," International Journal of Heat and Mass Transfer, vol. 50, no. 1314, pp. 2571-2581, 2007.

[20] Y. Zhu and S. Granick, "Rate-dependent slip of Newtonian liquid at smooth surfaces," Physical Review Letters, vol. 87, no. 9, Article ID 096105, pp. 961051-961054, 2001.

[21] Y. H. Wu, B. Wiwatanapataphee, and M. Hu, "Pressure-driven transient flows of Newtonian fluids through microtubes with slip boundary," Physica A, vol. 387, no. 24, pp. 5979-5990, 2008.

[22] B. Wiwatanapataphee, Y. H. Wu, M. Hu, and K. Chayantrakom, "A study of transient flows of Newtonian fluids through microannuals with a slip boundary," Journal of Physics A, vol. 42, no. 6, article 065206, 2009.

[23] G. C. Georgiou and G. Kaoullas, Newtonian Flow in a Triangularduct with Slip at the Wall, Mecanica, 2013.

[24] G. Kaoullas and G. C. Georgiou, Newtonian Poiseuille Flows with Slip and Non-Zero Slip Yield Stress, Mecanica, 2013.

[25] Y. H. Wu, B. Wiwatanapataphee, and X. Yu, "An enthalpy control volume method for transient mass and heat transport with solidification," International Journal of Computational Fluid Dynamics, vol. 18, no. 7, pp. 577-584, 2004.

[26] B. Wiwatanapataphee, "Modelling of non-Newtonian blood flow through stenosed coronary arteries," Dynamics of Continuous, Discrete \& Impulsive Systems B, vol. 15, no. 5, pp. 619-634, 2008.

[27] S. Amornsamankul, K. Kaorapapong, and B. Wiwatanapataphee, "Three-dimensional simulation of femur bone and implant in femoral canal using finite element method," International Journal of Mathematics and Computers in Simulation, vol. 4, no. 4, pp. 171-178, 2010.

[28] Y. H. Wu and B. Wiwatanapataphee, "Modelling of turbulent flow and multi-phase heat transfer under electromagnetic force," Discrete and Continuous Dynamical Systems B, vol. 8, no. 3, pp. 695-706, 2007.

[29] H.-B. Lee, I. W. Yeo, and K.-K. Lee, "Water flow and slip on NAPL-wetted surfaces of a parallel-walled fracture," Geophysical Research Letters, vol. 34, no. 19, Article ID L19401, 2007.

[30] B. Wiwatanapataphee, D. Poltem, Y. H. Wu, and Y. Lenbury, "Simulation of pulsatile flow of blood in stenosed coronary artery bypass with graft," Mathematical Biosciences and Engineering, vol. 3, no. 2, pp. 371-383, 2006.

[31] C. S. Man and Q. K. Sun, "On the significance of normal stress effects in the flow of glaciers," International Glaciological Society, pp. 268-273, 1987.

[32] C.-S. Man, "Nonsteady channel flow of ice as a modified secondorder fluid with power-law viscosity", Archive for Rational Mechanics and Analysis, vol. 119, no. 1, pp. 35-57, 1992. 


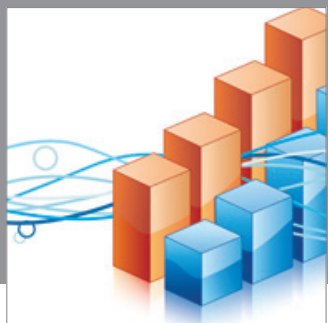

Advances in

Operations Research

mansans

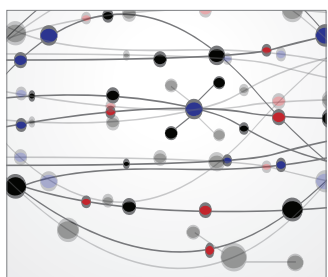

The Scientific World Journal
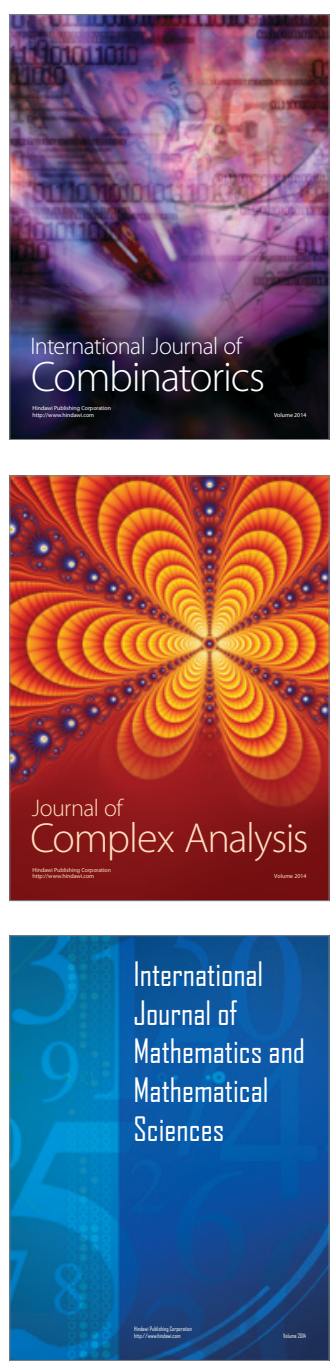
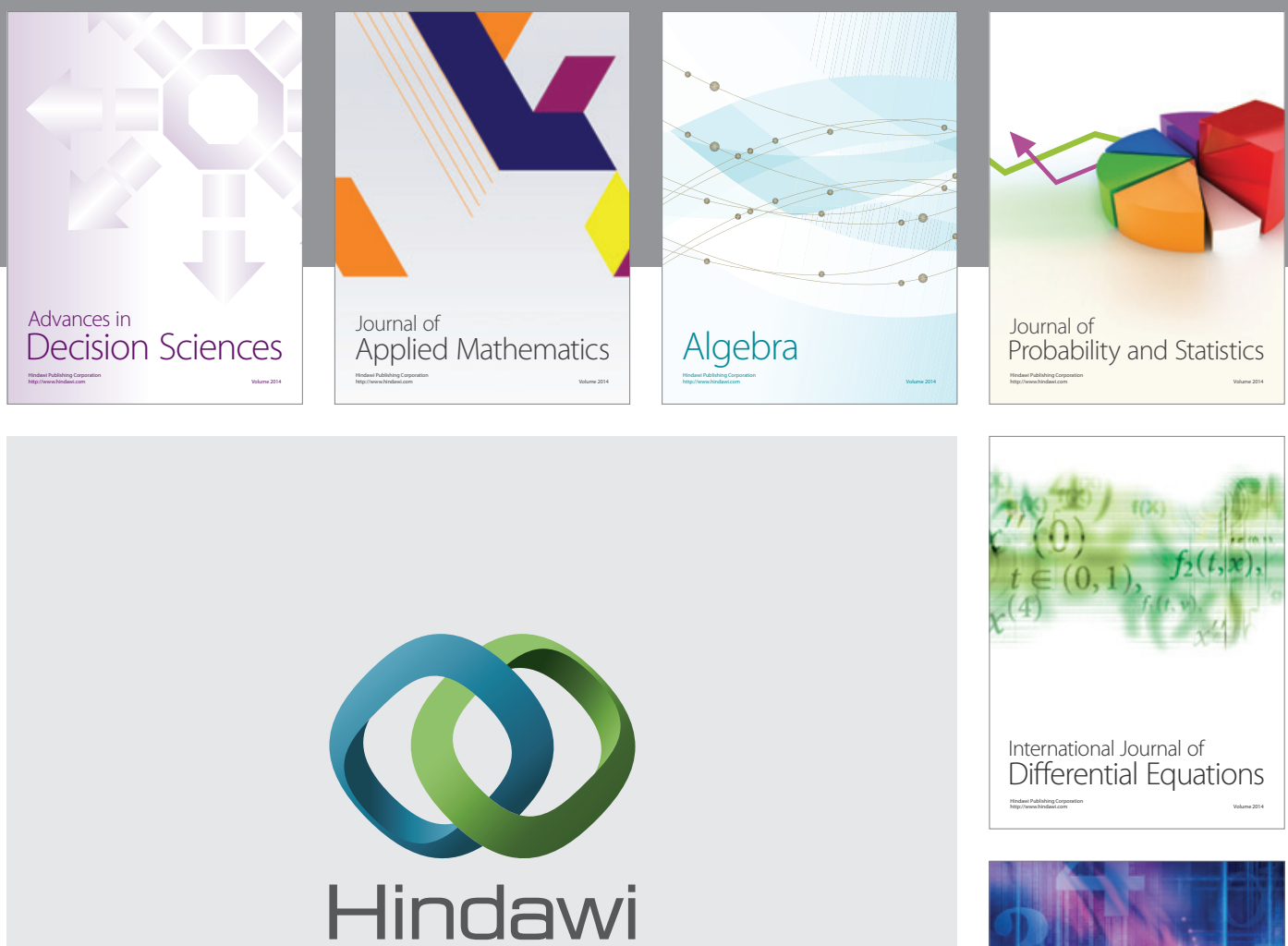

Submit your manuscripts at http://www.hindawi.com
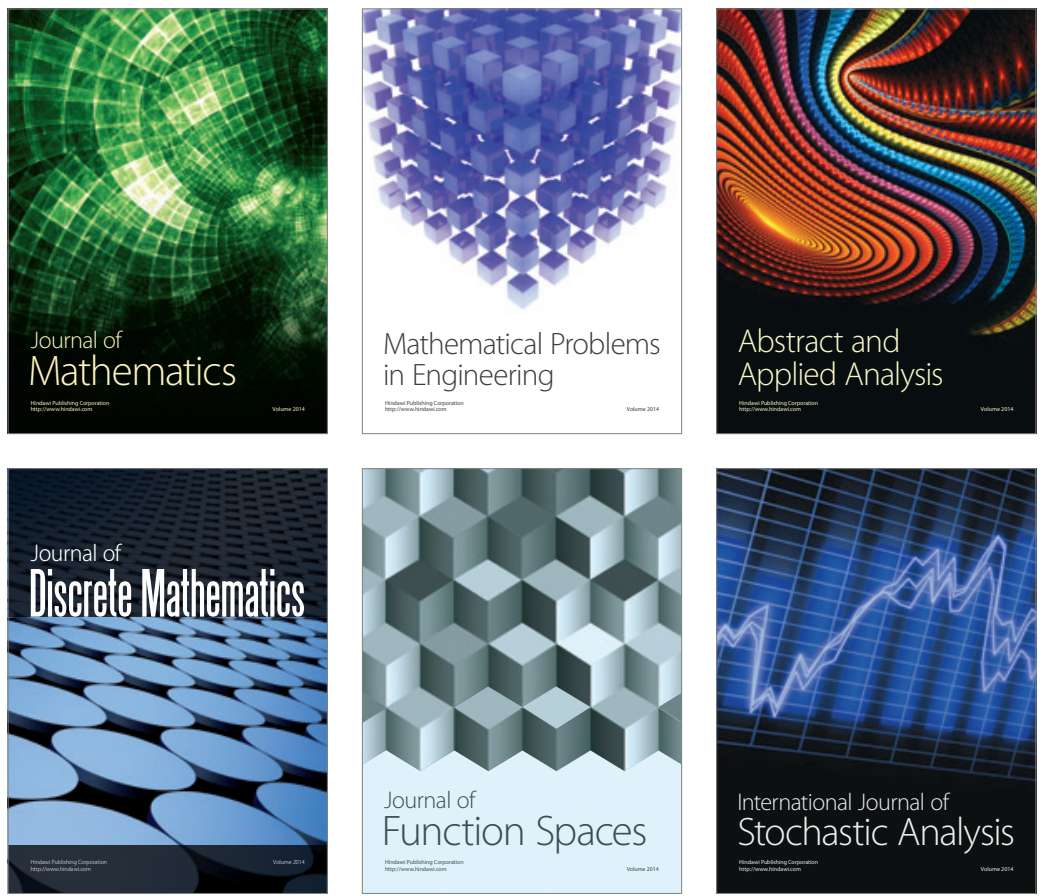

Journal of

Function Spaces

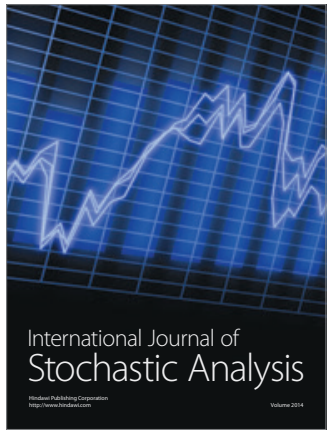

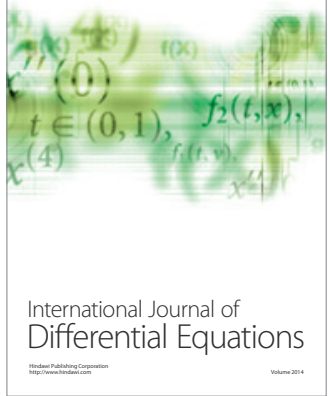
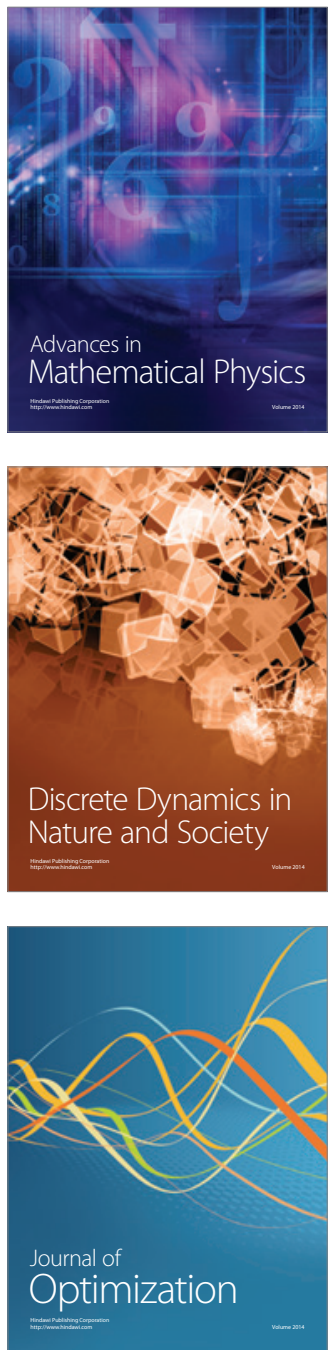